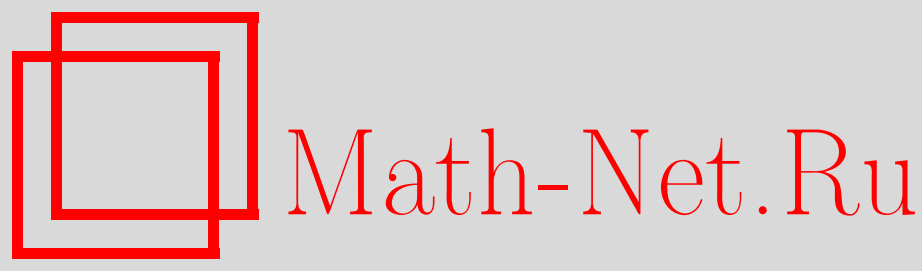

А. В. Зотов, А. М. Левин, М. А. Ольшанецкий, Ю. Б. Черняков, Квадратичные алгебры, связанные с эллиптическими кривыми, ТМФ, 2008, том 156, номер 2, 163-183

DOI: https://doi.org/10.4213/tmf6238

Использование Общероссийского математического портала Math-Net.Ru подразумевает, что вы прочитали и согласны с пользовательским соглашением http://www.mathnet.ru/rus/agreement

Параметры загрузки:

IP: 54.197 .217 .227

26 апреля 2023 г., 12:42:30

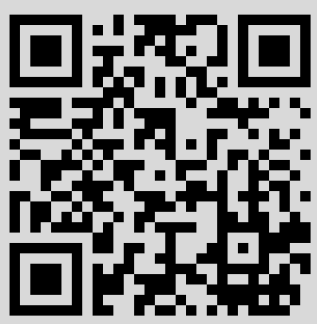




\title{
КВАДРАТИЧНЫЕ АЛГЕБРЫ, СВЯЗАННЫЕ С ЭЛЛИПТИЧЕСКИМИ КРИВЫМИ
}

\begin{abstract}
Построены квадратичные конечномерные пуассоновы алгебры, соответствующие векторному расслоению ранга $N$ и степени один над эллиптической кривой с $n$ отмеченными точками, и их квантовая версия. Алгебры параметризуются модулями кривых. При $N=2$ и $n=1$ они совпадают с алгебрами Склянина. Доказано, что пуассонова структура совместна со структурой Ли-Пуассона, определенной на прямой сумме $n$ копий $s l(N)$. Происхождение алгебр основано на пуассоновой редукции канонических скобок на аффинном пространстве над кокасательным расслоением к группам автоморфизмов векторных расслоений.
\end{abstract}

Ключевые слова: пуассонова структура, интегрируемые системы.

\section{1. ВВЕДЕНИЕ}

В настоящей работе построены квадратичные пуассоновы алгебры (классические алгебры Склянина-Фейгина-Одесского), основанные на перестановочных соотношениях с эллиптической $s l(N, \mathbb{C}) r$-матрицей Белавина-Дринфельда [1], и их квантовая версия, соответствующая вертексным эллиптическим матрицам [2]. Эти алгебры параметризованы модулями пространства комплексных структур эллиптических кривых с $n$ отмеченными точками, а в квантовом случае - постоянной Планка, соответствующей кривой. При $N=2$ и $n=1$ мы приходим к алгебре Склянина [3]. Построенные алгебры являются частным случаем общей конструкции [4], но в отличие от общего случая они конечнопорожденные. Мы описываем явным образом скобки Пуассона между генераторами и соответствующие квадратичные соотношения в квантовом случае в терминах квазипериодических функций на пространстве модулей. С одной стороны, в классическом случае пуассоновы алгебры имеют вид квадратичных алгебр на прямом произведении $n$ копий $G L(N, \mathbb{C})$ с нетривиальным смешиванием компонент. С другой стороны, существует стандартная линейная

* Институт теоретической и экспериментальной физики, Москва, Россия.

E-mail: chernyakov@itep.ru, olshanet@itep.ru

${ }^{\dagger}$ Институт океанологии им. П. П. Ширшова РАН, Москва, Россия.

E-mail: alevin@wave.sio.rssi.ru

${ }^{\ddagger}$ Max Planck Institute, Bonn, Germany. E-mail: zotov@itep.ru 
структура Ли-Пуассона на прямой сумме $n$ копий $G L(N, \mathbb{C})$-значных групп Ли. Мы доказываем совместность этих двух структур.

Классические алгебры определяют симметрии эллиптических обобщений систем Шлезингера и Гарнье [5], [6].

В разделе 2 выводится с помощью пуассоновой редукции классическая вертексная $r$-матрица и $G L(N, \mathbb{C})$-значная матрица Лакса с $n$ простыми полюсами из канонических скобок на некотором обобщении кокасательного расслоения двухпетлевой группы $G L(N, \mathbb{C})$. В разделе 3 мы получаем точный вид скобок и доказываем, что они совместны со скобками Ли-Пуассона. Раздел 4 посвящен квантовому случаю.

\section{2. КЛАССИЧЕСКИЕ КОММУТАЦИОННЫЕ СООТНОШЕНИЯ ДВУХПЕТЛЕВОЙ ГРУППЫ $G L(N, \mathbb{C})$}

2.1. Расслоения степени один над эллиптическими кривыми. Пусть $\Sigma_{\tau}=\mathbb{C} /(\mathbb{Z}+\tau \mathbb{Z})$ - эллиптическая кривая с модулярным параметром $\tau, \operatorname{Im} \tau>0$. Рассмотрим векторное расслоение $E_{N}$ ранга $N$ над $\Sigma_{\tau}$. Оно описывается своими сечениями $s=\left(s_{1}(z, \bar{z}), \ldots, s_{N}(z, \bar{z})\right)$ с монодромиями

$$
s^{\mathrm{T}}(z+1, \bar{z}+1)=Q s^{\mathrm{T}}(z, \bar{z}), \quad s^{\mathrm{T}}(z+\tau, \bar{z}+\bar{\tau})=\widetilde{\Lambda} s^{\mathrm{T}}(z, \bar{z}),
$$

где

$$
Q=\operatorname{diag}\left(1, \mathbf{e}_{N}, \ldots, \mathbf{e}_{N}^{N-1}\right), \quad \mathbf{e}_{N}=e^{2 \pi i / N}, \quad \widetilde{\Lambda}=\mathbf{e}_{N}^{-(z+\tau / 2)} \Lambda, \quad \Lambda=\left(E_{j, j+1}\right),
$$

где $E_{j, j+1}-$ матрица с единицей на $(j, j+1)$-ом месте. Поскольку $\operatorname{det} Q= \pm 1$ и $\operatorname{det} \widetilde{\Lambda}= \pm \mathbf{e}_{1}^{-(z+\tau / 2)}$, детерминанты матриц сдвига имеют те же квазипериоды, что и тета-функции Якоби. Тета-функции имеют простые полюсы в фундаментальном параллелограмме периодов $\Sigma_{\tau}$. Таким образом, векторное расслоение $E_{N}$ имеет степень один.

Выберем голоморфное сечение $\bar{\partial} s=0$ в виде

$$
s(z)=\left(\theta\left[\begin{array}{c}
\frac{1}{N} \\
0
\end{array}\right](z ; N \tau), \ldots, \theta\left[\begin{array}{l}
1 \\
0
\end{array}\right](z ; N \tau)\right) .
$$

Зададим преобразования $s^{\mathrm{T}} \rightarrow f(z, \bar{z}) s^{\mathrm{T}}$ гладкими отображениями

$$
f: \Sigma_{\tau} \rightarrow G L(N, \mathbb{C}), \quad f \in \Omega_{C^{\infty}}^{(0,0)}\left(\Sigma_{\tau}, G L(N, \mathbb{C})\right),
$$

с монодромиями

$$
f(z+1, \bar{z}+1)=Q^{-1} f(z, \bar{z}) Q, \quad f(z+\tau, \bar{z}+\bar{\tau})=\widetilde{\Lambda}^{-1} f(z, \bar{z}) \widetilde{\Lambda} .
$$

Эти преобразования сохраняют степень $E_{N}$ и поэтому порождают калибровочную группу $\mathcal{G}=\{f(z, \bar{z})\}$ расслоения $E_{N}$.

В общем случае операторы

$$
d_{\bar{A}}=\bar{\partial}+\bar{A}: \Omega^{(0,0)}\left(\Sigma_{\tau}, E_{N}\right) \rightarrow \Omega^{(0,1)}\left(\Sigma_{\tau}, E_{N}\right)
$$


определяют комплексную структуру $E_{N}$. Сечение голоморфное, если $d_{\bar{A}}\left(s^{\mathrm{T}}\right)=0$. Здесь мы предполагаем, что оператор $\bar{A}$ имеет те же монодромии, что и сечения расслоения $E_{N}$ :

$$
\bar{A}(z+1, \bar{z}+1)=Q^{-1} \bar{A}(z, \bar{z}) Q, \quad \bar{A}(z+\tau, \bar{z}+\bar{\tau})=\widetilde{\Lambda}^{-1} \bar{A}(z, \bar{z}) \widetilde{\Lambda} .
$$

Две комплексные структуры, определенные связностями $\bar{A}$ и $\bar{A}^{f}$, называются эквивалентными, если они относятся к калибровочному преобразованию

$$
\bar{A}^{f}=f^{-1} \bar{A} f+f^{-1} \bar{\partial} f, \quad f \in \mathcal{G} .
$$

Факторпространство порожденных связностей $\mathcal{A}=\{\bar{A}\}$ по действию $\mathcal{G}$ является пространством модулей голоморфных расслоений $\operatorname{Bun}\left(E_{N}\right)=\mathcal{A} / \mathcal{G}$.

Рассмотрим двухпетлевую группу $L L(G L(N, \mathbb{C}))$, представленную пространством сечений $\{g(z, \bar{z})\}=\Omega_{C^{\infty}}^{(0,0)}\left(\Sigma_{\tau}, G L(N, \mathbb{C})\right)$ с монодромиями

$$
g(z+1, \bar{z}+1)=Q^{-1} g(z, \bar{z}) Q, \quad g(z+\tau, \bar{z}+\bar{\tau})=\widetilde{\Lambda}^{-1} g(z, \bar{z}) \widetilde{\Lambda} .
$$

Двухпетлевая группа $L L(G L(N, \mathbb{C}))$ с этими условиями квазипериодичности является группой автоморфизмов Aut $E_{N}$ векторного расслоения $E_{N}$ степени один.

2.2. Пуассонова структура на $\mathcal{R}$. На пространстве

$$
\mathcal{R}=\mathcal{A} \times \Omega_{C^{\infty}}^{(0,0)}\left(\Sigma_{\tau}, G L(N, \mathbb{C})\right)=\{(\bar{\partial}+\bar{A}, g)\}
$$

имеется симплектическая форма

$$
\omega=\int_{\Sigma_{\tau}} K\left\langle d\left(\bar{A} g^{-1}\right) \wedge d g\right\rangle+\frac{1}{2} \int_{\Sigma_{\tau}} K\left\langle g^{-1} d g \wedge \bar{\partial}\left(g^{-1} d g\right)\right\rangle,
$$

где $\langle\cdot\rangle$ - след в векторном представлении, $K$ - сечение канонического расслоения над $\Sigma_{\tau}, K \in \Omega^{(1,0)}\left(\Sigma_{\tau}\right)$; мы выбираем $K=d z$. Пространство $\mathcal{R}$ является аффинным пространством над кокасательным расслоением к двухпетлевой группе $T^{*}(L L(G L(N, \mathbb{C})))$.

Преобразования (2.2) вместе с преобразованием

$$
g \rightarrow f^{-1} g f
$$

являются каноническими относительно симплектической формы (2.4). Гамильтоновы векторные поля $V_{\epsilon}, \epsilon \in \operatorname{Lie}(\mathcal{G})$, на $\mathcal{R}\left(V_{\epsilon} \omega=d \mu^{*}\right)$ порождены гамильтонианом

$$
\mu^{*}(\epsilon ; \bar{A}, g)=\int_{\Sigma_{\tau}} K\left\langle\epsilon\left(g \bar{A} g^{-1}-\bar{\partial} g g^{-1}-\bar{A}\right)\right\rangle \text {. }
$$

ЗАмечание 1. Пусть $\Phi \in \Omega_{C^{\infty}}^{(1,0)}\left(\Sigma_{\tau}\right.$, End $\left.E_{N}\right)$ - поле Хиггса и $g=e^{\hbar K^{-1} \Phi}$, где $\hbar \in \mathbb{C}$. В пределе $\hbar \rightarrow 0$

$$
g \sim K^{-1}(\operatorname{Id}+\hbar \Phi+\cdots) .
$$

Форма (2.4) в первом порядке становится канонической формой на расслоении Хиггса $\left\{\left(d_{\bar{A}}, \Phi\right)\right\}$. Симметрии определяют гамильтониан

$$
\mu^{*}(\epsilon ; \bar{A}, \Phi)=\int_{\Sigma_{\tau}}\langle\epsilon(\bar{\partial} \Phi+[\bar{A}, \Phi])\rangle .
$$

Таким образом, $\mathcal{R}$ есть деформация расслоения Хиггса. 
Обращение формы (2.4) определяет пуассонову структуру на $\mathcal{R}$. В координатных терминах в базисе (Б.1) $\bar{A}=\sum_{\alpha \in \widetilde{\mathbb{Z}}_{N}^{(2)}} \bar{A}_{\alpha} T_{\alpha}, g=\sum_{a \in \mathbb{Z}_{N}^{(2)}} g_{a} T_{a}$, и обращение формы (2.4) принимает вид

$$
\begin{gathered}
K\left\{\bar{A}_{\alpha}(z, \bar{z}), \bar{A}_{\beta}(w, \bar{w})\right\}=C_{\alpha+\beta} \bar{A}_{\alpha+\beta} \delta(z-w, \bar{z}-\bar{w})+\bar{\partial} \delta(z-w, \bar{z}-\bar{w}) \delta_{\alpha,-\beta}, \\
K\left\{g_{a}(z, \bar{z}), \bar{A}_{\beta}(w, \bar{w})\right\}=\mathbf{e}_{N}^{(a \times \beta)} g_{a+\beta}(z, \bar{z}) \delta(z-w, \bar{z}-\bar{w}) \\
\left\{g_{a}(z, \bar{z}), g_{b}(w, \bar{w})\right\}=0 .
\end{gathered}
$$

Скобки определяют пуассонову алгебру $\mathcal{O}(\mathcal{R})$ с группой симметрии $\mathcal{G}$.

Определим пуассонову подалгебру $\mathbf{P}_{\Sigma_{\tau}}$ пуассоновой алгебры $\mathcal{O}(\mathcal{R})$. Она удовлетворяет следующим условиям:

1) связность $\bar{A}$ принимает значения в подалгебре $s l(N, \mathbb{C}), g$ принимает значение в $G L(N, \mathbb{C})$;

2) $\mathbf{P}_{\Sigma_{\tau}}$ генерируется голоморфными функционалами над $\mathcal{R}$ с пробной функцией, исчезающей в точке $z=0$.

Подалгебра $\mathbf{P}_{\Sigma_{\tau}}$ имеет центр $\mathcal{Z}$, порожденный $\operatorname{det} g(z, \bar{z})$. Группа симметрий $\mathcal{G}^{\mathrm{s}} \subset$ $\mathcal{G}$ подалгебры $\mathbf{P}_{\Sigma_{\tau}}$ порождена гладкими отображениями $f: \Sigma_{\tau} \rightarrow S L(N, \mathbb{C})$.

2.3. Пуассонова редукция. Наша цель - вычислить пуассонову структуру, редуцированную по действию $\mathcal{G}^{\mathrm{s}}$.

Стандартная пуассонова редукция $\mathbf{P}_{\Sigma_{\tau}}^{\text {red }}$ подалгебры $\mathbf{P}_{\Sigma_{\tau}}$ описывается следующим образом. Пусть $\mathbf{P}_{\Sigma_{\tau}}^{\mathcal{G}^{\mathrm{s}}}$ - инвариантная пуассонова подалгебра, $I^{\mathcal{G}^{\mathrm{s}}}=\left\{\mu^{*}(\epsilon) F(\bar{A}, g) \mid\right.$ $\left.F(\bar{A}, g) \in \mathbf{P}_{\Sigma_{\tau}}\right\}$ является идеалом в $\mathbf{P}_{\Sigma_{\tau}}^{\mathcal{G}^{\mathrm{s}}}$, порожденным функционалом $\mu^{*}(\epsilon)(2.6)$, где $\epsilon \in \operatorname{Lie}\left(\mathcal{G}^{\mathrm{s}}\right)$. Редуцированная пуассонова алгебра $\mathbf{P}_{\Sigma_{\tau}}^{\text {red }}$ есть фактор-алгебра:

$$
\mathbf{P}_{\Sigma_{\tau}}^{\text {red }}=\mathbf{P}_{\Sigma_{\tau}}^{\mathcal{G}^{\mathrm{s}}} / I^{\mathcal{G}^{\mathrm{s}}}:=\mathbf{P}_{\Sigma_{\tau}} / / \mathcal{G}^{\mathrm{s}}
$$

В нашей конструкции используется другой идеал $\mathbf{P}_{\Sigma_{\tau} \mathcal{G}^{\text {s }}}$, который будет определен ниже.

Прежде всего, вычислим скобки в инвариантной подалгебре $\mathbf{P}_{\Sigma_{\tau}^{\mathcal{G}^{\mathrm{s}}}}$. Вследствие условия на монодромию (2.1), порожденное поле $\bar{A}$ калибровочно-эквивалентно тривиальному полю $f^{-1} \bar{A} f+f^{-1} \bar{\partial} f=0$, поэтому

$$
\bar{A}=-\bar{\partial} f[\bar{A}] f^{-1}[\bar{A}] .
$$

Отметим, что монодромии калибровочных матриц (2.3) "предохраняют" от нетривиальных остаточных симметрий. Пусть $f[\bar{A}](z, \bar{z})$ - решение уравнения (2.12). Рассмотрим преобразование $g$ под действием решения (2.12):

$$
L[\bar{A}, g](z, \bar{z})=f[\bar{A}](z, \bar{z}) g(z, \bar{z}) f^{-1}[\bar{A}](z, \bar{z}) .
$$

Калибровочно-инвариантная подалгебра $\mathbf{P}_{\Sigma_{\tau}}^{\mathcal{G}^{\mathrm{s}}}$ порождена матрицами $L$ :

$$
\mathbf{P}_{\Sigma_{\tau}}^{\mathcal{G}^{\mathrm{s}}}=\{\Psi(\bar{A}, g)=\Psi(0, L)\}
$$

ПРЕДЛОЖЕНИЕ 1. СКобки на $\mathbf{P}_{\Sigma_{\tau}^{\mathcal{G}}}^{\mathcal{G}^{\mathrm{s}}}$ принимают форму классических коммутационных соотношений

$$
\left\{L_{1}(z, \bar{z}), L_{2}(w, \bar{w})\right\}=\left[r(z-w), L_{1}(z, \bar{z}) \otimes L_{2}(w, \bar{w})\right],
$$


где $L_{1}(z, \bar{z})=L(z, \bar{z}) \otimes \mathrm{Id}, L_{2}(w, \bar{w})=\mathrm{Id} \otimes L(w, \bar{w}), r(z, w)$ есть классическая эллиптическая г-матрица Белавина-Дринфельда [1].

ДокАЗАТЕЛЬство. Вычисление скобок в $\mathbf{P}_{\Sigma_{\tau}}^{\mathcal{G}^{\text {s }}}$ сводится к вычислениям скобок Пуассона на поверхности уровня $\bar{A}=0, f=\mathrm{Id}$ между матричными элементами (2.13) с помощью (2.8)-(2.10). Для этого нам нужны только выражения

$$
r_{\alpha, \beta}\left(z, \bar{z} ; z^{\prime}, \bar{z}^{\prime}\right)=\left.\frac{\delta f_{\alpha}(z, \bar{z})}{\delta \bar{A}_{\beta}\left(z^{\prime}, \bar{z}^{\prime}\right)}\right|_{\bar{A}=0} .
$$

Прямые вычисления скобок $\{L, L\}$, представленные в [7], сводятся к искомой $r$-матричной форме (2.14).

Найдем $r$-матрицу. Вследствие уравнения (2.12) $r$ есть функция Грина оператоpa $\bar{\partial}$

$$
\bar{\partial} r_{\alpha, \beta}\left(z, \bar{z} ; z^{\prime}, \bar{z}^{\prime}\right)=\delta_{\alpha+\beta, 0} \delta\left(z-z^{\prime}, \bar{z}-\bar{z}^{\prime}\right)
$$

имеющая следующие квазипериоды:

$$
\begin{aligned}
& r(z+1, \bar{z}+1)=\left(Q^{-1} \otimes \mathrm{Id}\right) r(z, \bar{z})(Q \otimes \mathrm{Id}), \\
& r(z+\tau, \bar{z}+\bar{\tau})=\left(\Lambda^{-1} \otimes \mathrm{Id}\right) r(z, \bar{z})(\Lambda \otimes \mathrm{Id}) .
\end{aligned}
$$

Из (2.16) следует, что $r_{\alpha, \beta}$ - мероморфная и сингулярная функция на диагонали,

$$
\lim _{z^{\prime} \rightarrow z} r_{\alpha, \beta}\left(z, z^{\prime}\right)=\frac{1}{z-z^{\prime}} T_{\alpha} \otimes T_{\beta} \delta_{\alpha+\beta, 0}
$$

Учитывая (А.5), (Б.5), (Б.7), получаем

$$
r(z, w)=r(z-w)=\sum_{\alpha} \varphi_{\alpha}(z-w) T_{\alpha} \otimes T_{-\alpha} .
$$

Это и есть классическая $r$-матрица Белавина-Дринфельда [1]. Эта матрица удовлетворяет классическому уравнению Янга-Бакстера, которое обеспечивает выполнение тождества Якоби для скобок (2.14).

ЗАмЕчАние 2. Если имеет место (2.7), то только нетривиальные скобки (2.9) допускают форму

$$
\left\{\Phi_{\alpha}(z, \bar{z}), \bar{A}_{\beta}(w, \bar{w})\right\}=\delta_{\alpha,-\beta} \delta(z-w, \bar{z}-\bar{w})
$$

a (2.14) заменяется на линейные скобки

$$
\left\{L_{1}(z, \bar{z}), L_{2}(w, \bar{w})\right\}=\left[r(z-w), L(z, \bar{z}) \otimes \operatorname{Id}+\operatorname{Id} \otimes \operatorname{Id} L_{2}(w, \bar{w})\right] .
$$

Зафиксируем дивизор $D_{n}$ в несовпадающих точках на $\Sigma_{\tau}$ :

$$
D_{n}=\left(x_{1}, \ldots, x_{n}\right), \quad x_{j} \neq x_{k}, \quad x_{j} \in \Sigma_{\tau} .
$$

Определим подалгебру $\operatorname{Lie}\left(D_{n}\right)\left(\mathcal{G}^{\mathrm{s}}\right) \subset \operatorname{Lie}\left(\mathcal{G}^{\mathrm{s}}\right)$ :

$$
\operatorname{Lie}\left(D_{n}\right)\left(\mathcal{G}^{\mathrm{s}}\right)=\left\{\varepsilon \in \operatorname{Lie}\left(\mathcal{G}^{\mathrm{s}}\right) \mid \varepsilon\left(x_{j}, \bar{x}_{j}\right)=0, x_{j} \in D_{n}\right\} .
$$


Рассмотрим идеал $I\left(D_{n}\right)$, порожденный функционалом

$$
\mu_{D_{n}}^{*}(\varepsilon ; \bar{A}, g)=\mu_{D_{n}}^{*}(\varepsilon ; L)=\int_{\Sigma_{\tau}}\langle\varepsilon \bar{\partial} L(z, \bar{z})\rangle
$$

где $\varepsilon$ принадлежит подалгебре $\operatorname{Lie}\left(\mathcal{G}^{\mathrm{s}}\left(D_{n}\right)\right)$. Поскольку функционал $\mu_{D_{n}}^{*}$ зависит только от $L$, то $I\left(D_{n}\right) \subset \mathbf{P}_{\Sigma_{\tau}}^{\mathcal{G}^{\mathrm{s}}}$.

Рассмотрим факторизованную пуассонову алгебру $\mathbf{P}_{\Sigma_{\tau}}^{\mathcal{G}^{\mathrm{s}}} / I\left(D_{n}\right)$.

ПРЕДЛОЖЕНИЕ 2. Редуиированная пуассонова алгебра

$$
\mathbf{P}_{\Sigma_{\tau}, D_{n}}^{\text {red }}=\mathbf{P}_{\Sigma_{\tau}}^{\mathcal{G}^{\mathrm{s}}} / I\left(D_{n}\right)
$$

является финитно порожденной, $\operatorname{dim} \mathbf{P}_{\Sigma_{\tau}, D_{n}}^{\mathrm{red}}=n N^{2}$. Maтрица $L(z)$ в классических коммутационных соотношениях (матрица Лакса) принимает вид

$$
L=S_{0} T_{0}+\sum_{j=1}^{n}\left(S_{0}^{j} E_{1}\left(z-x_{j}\right) T_{0}+\widetilde{L}_{j}\right), \quad \widetilde{L}_{j}=\sum_{\alpha} S_{\alpha}^{j} \varphi_{\alpha}\left(z-x_{j}\right) T_{\alpha},
$$

где

$$
\sum_{j=1}^{n} S_{0}^{j}=0
$$

функиии $\varphi_{\alpha}\left(z-x_{j}\right)$ определень в (Б.5), $E_{1}\left(z-x_{j}\right)$ есть первая функиия в базисе Эйзенштейна (А.1).

ДокАЗАТЕльство. Проанализируем решения уравнения

$$
\mu_{D_{n}}^{*}(\varepsilon ; L)=\int_{\Sigma_{\tau}}\langle\varepsilon \bar{\partial} L(z, \bar{z})\rangle=0 .
$$

Решения являются мероморфными квазипериодическими отображениями, имеющими простые полюсы в отмеченных точках. Пусть $L(z)=\sum_{a} L_{a}(z) T_{a}$ есть разложение $L$ по базису $T_{a}$ группы $G L(N, \mathbb{C})$. Из $(2.3)$ следует, что

$$
L_{a}(z+1)=\mathbf{e}_{N}^{a_{2}} L_{a}(z), \quad L_{a}(z+\tau)=\mathbf{e}_{N}^{-a_{1}} L_{a}(z), \quad a=\left(a_{1}, a_{2}\right) .
$$

Функции $\varphi_{a}\left(z-x_{j}\right)\left(\right.$ Б.5) имеют эти монодромии (Б.7) и простые полюсы в $x_{j}$. Они определяют $n$-мерный базис в пространстве квазипериодических функций (Б.13) с полюсами в $x_{j}$. Если $a=(0,0)$, то $L_{0}(z)$ является двоякопериодической функцией с простыми полюсами в $x_{j}$. Базисом в этом пространстве являются единица и функции Эйзенштейна $E_{1}\left(z-x_{j}\right)$, у которых сумма вычетов равна нулю. Таким образом, пространство имеет размерность $n$ и выполняются соотношения (2.21) и (2.22).

Как мы упоминали выше, $\operatorname{det} g$ порождают функционалы Казимира в $\mathbf{P}_{\Sigma_{\tau}}^{\text {red }}$. Вследствие этого скобки на $\mathbf{P}_{\Sigma_{\tau}, D_{n}}^{\text {red }}$ вырожденны. Функции $\operatorname{det} L(z)$ являются производящими функциями для элементов Казимира $C^{\mu}(j)$. Поскольку $\operatorname{det} L(z)$ является двоякопериодической функцией, он может быть разложен по базису эллиптических функций (А.3):

$$
\operatorname{det} L(z)=C^{0}+\sum_{j}^{n} C^{1}(j) E_{1}\left(z-x_{j}\right)+C^{2}(j) E_{2}\left(z-x_{j}\right)+\cdots+C^{N}(j) E_{N}\left(z-x_{j}\right) .
$$


Вследствие условия

$$
\sum_{j=1}^{n} C^{1}(j)=0
$$

количество независимых элементов Казимира равно $N n$. Симплектический лист общего положения

$$
\mathcal{R}_{n, N}^{2}=\mathbf{P}_{\Sigma_{\tau}, D_{n}}^{\text {red }} /\left\{\left(C^{\mu}(j)=C(j)_{(0)}^{\mu}\right), \mu=1, \ldots, N, j=1, \ldots, N\right\}
$$

имеет размерность

$$
\operatorname{dim}\left(\mathcal{R}_{n, N}^{2}\right)=n N(N-1)
$$

Заметим, что эта величина совпадает с суммой размерностей $n$ коприсоединенных орбит $G L(N, \mathbb{C})$ общего положения.

\section{3. СТРУКТУРА РЕДУЦИРОВАННОГО ПУАССОНОВА ПРОСТРАНСТВА}

3.1. Точный вид квадратичных скобок. Предложение 2 дает редуцированную пуассонову алгебру $\mathbf{P}_{\Sigma_{\tau}, D_{n}}^{\text {red }}$ с генераторами

$$
\left\{S_{0},\left(S_{0}^{j}, \mathbf{S}^{j}=\left\{S_{\alpha}^{j}\right\}, j=1, \ldots, n\right) \mid \sum_{j=1}^{n} S_{0}^{j}=0\right\} .
$$

Скобки между генераторами вычислены в [6].

ПрЕДЛОЖЕНИЕ 3. СКобки Пуассона на пространстве $\mathbb{C}^{n N^{2}}$ в терминах генераторов (3.1) принимают вид $\left.{ }^{1}\right)$

$$
\begin{aligned}
&\left\{S_{0}, S_{0}^{j}\right\}_{2}=\left\{S_{0}^{j}, S_{0}^{k}\right\}_{2}=\left\{S_{\alpha}^{j}, S_{\alpha}^{k}\right\}_{2}=0 \\
&\left\{S_{0}, S_{\alpha}^{k}\right\}_{2}= \sum_{\gamma \neq \alpha} \mathbf{C}(\alpha, \gamma)\left(S_{\alpha-\gamma}^{k} S_{\gamma}^{k} E_{2}(\breve{\gamma})-\sum_{j \neq k} S_{-\gamma}^{j} S_{\alpha+\gamma}^{k} f_{\gamma}\left(x_{k}-x_{j}\right)\right) \\
&\left\{S_{\alpha}^{k}, S_{\beta}^{k}\right\}_{2}=\mathbf{C}(\alpha, \beta) S_{0} S_{\alpha+\beta}^{k}+\sum_{\gamma \neq \alpha, \beta} \mathbf{C}(\gamma, \alpha-\beta) S_{\alpha-\gamma}^{k} S_{\beta+\gamma}^{k} \mathbf{f}_{\alpha, \beta, \gamma}+ \\
& \quad+\mathbf{C}(\alpha, \beta) S_{0}^{k} S_{\alpha+\beta}^{k}\left(E_{1}(\breve{\alpha}+\breve{\beta})-E_{1}(\breve{\alpha})-E_{1}(\breve{\beta})\right)- \\
& \quad-\mathbf{C}(\alpha, \beta) \sum_{j \neq k}\left[S_{0}^{k} S_{\alpha+\beta}^{j} \varphi_{\alpha+\beta}\left(x_{k}-x_{j}\right)-S_{0}^{j} S_{\alpha+\beta}^{k} E_{1}\left(x_{k}-x_{j}\right)\right]- \\
& \quad-2 \sum_{j \neq k} \mathbf{C}(\gamma, \alpha-\beta) S_{\alpha-\gamma}^{k} S_{\beta+\gamma}^{k} \varphi_{\beta+\gamma}\left(x_{k}-x_{j}\right),
\end{aligned}
$$

где величины $\mathbf{f}_{\alpha, \beta, \gamma}, E_{2}(\breve{\alpha}), E_{1}(\breve{\alpha})$ определенъ в (Б.12) и (Б.4). При $j \neq k$

$$
\begin{aligned}
\left\{S_{\alpha}^{j}, S_{\beta}^{k}\right\}_{2}= & \sum_{\gamma \neq \alpha,-\beta} \mathbf{C}(\gamma, \alpha-\beta) S_{\alpha-\gamma}^{j} S_{\beta+\gamma}^{k} \varphi_{\gamma}\left(x_{j}-x_{k}\right)- \\
& \quad-\mathbf{C}(\alpha, \beta)\left(S_{0}^{j} S_{\alpha+\beta}^{k} \varphi_{\alpha}\left(x_{j}-x_{k}\right)-S_{0}^{k} S_{\alpha+\beta}^{j} \varphi_{-\beta}\left(x_{k}-x_{j}\right)\right),
\end{aligned}
$$

1) Индекс 2 у фигурных скобок $\{\cdot, \cdot\}_{2}$ означает квадратичные скобки. 


$$
\left\{S_{0}^{j}, S_{\beta}^{k}\right\}_{2}= \begin{cases}2 \sum_{\gamma} \mathbf{C}(\gamma,-\beta) S_{-\gamma}^{j} S_{\beta+\gamma}^{k} \varphi_{\gamma}\left(x_{k}-x_{j}\right), & j \neq k, \\ -2 \sum_{m \neq k} \sum_{\gamma} \mathbf{C}(\gamma,-\beta) S_{-\gamma}^{k} S_{\beta+\gamma}^{m} \varphi_{\beta+\gamma}\left(x_{k}-x_{m}\right), & j=k .\end{cases}
$$

Эта алгебра является точной специальной формой общей конструкции квадратичных пуассоновых алгебр [4]. При $n=1$ эта алгебра была вычислена в [8]; при $n=1$ и $N=2$ она является классической алгеброй Склянина [3].

3.2. Твистованные расслоения. Нам понадобится эквивалентная форма пуассоновой алгебры на $\mathbb{C}^{n N^{2}}$. Рассмотрим твистованное расслоение $E_{N}^{\prime}=\operatorname{Aut}\left(E_{N}\right) \otimes \mathcal{L}$, где $\mathcal{L}$ - тривиальное линейное расслоение над $\Sigma_{\tau}$. Сечения $E_{N}^{\prime}$ являются сечениями $E_{N}$, умноженными на $\vartheta(z+\eta) / \vartheta(z), \eta \in \Sigma_{\tau}$. Поэтому функции сдвигов $E_{N}^{\prime}$ имеют следующий вид:

$$
\operatorname{ad}(Q): z \rightarrow z+1, \quad e^{-2 \pi i \eta} \operatorname{ad}(\widetilde{\Lambda}): z \rightarrow z+\tau .
$$

Из (Б.6), (Б.8) следует, что решением уравнения (2.23) с этими монодромиями и простыми полюсами дивизора $\widetilde{D}_{n}=\left(\tilde{x}_{1}, \ldots, \tilde{x}_{n}\right)$ является

$$
L_{\widetilde{D}_{n}}^{\eta}=\sum_{j=1}^{n}\left[\widetilde{S}_{0}^{j} \varphi_{\eta}\left(z-\tilde{x}_{j}\right) T_{0}+\sum_{\alpha} \widetilde{S}_{\alpha}^{j} \varphi_{\alpha, \eta}\left(z-\tilde{x}_{j}\right) T_{\alpha}\right]
$$

где $\varphi_{\eta}=\varphi_{0, \eta}$. Соответствующая алгебра $\widetilde{\mathbf{P}}_{\Sigma_{\tau}, \widetilde{D}_{n}}^{\text {red }}$ определена, как и выше, классическими перестановочными соотношениями

$$
\left\{L_{1, \widetilde{D}_{n}}^{\eta}(z), L_{2, \widetilde{D}_{n}}^{\eta}(w)\right\}=\left[r(z-w), L_{1, \widetilde{D}_{n}}^{\eta}(z) \otimes L_{2, \widetilde{D}_{n}}^{\eta}(w)\right]
$$

с набором $n N^{2}$ генераторов:

$$
\widetilde{\mathbf{S}}^{j}=\left\{\widetilde{S}_{a}^{j}\right\}, \quad a=\left(a_{1}, a_{2}\right) \in \mathbb{Z}_{N}^{(2)}, \quad j=1, \ldots, n .
$$

Скобки между генераторами могут быть получены из (3.8), как и раньше. Нам не потребуются точные соотношения, потому что мы сразу докажем эквивалентность этих двух алгебр. В разделе 4 рассмотрим только скобки, содержащие $\widetilde{S}_{0}^{k}$ в правой части (ср. (3.4) и (3.5)),

$$
\begin{aligned}
\left\{\widetilde{S}_{\alpha}^{k}, \widetilde{S}_{\beta}^{k}\right\}= & \sum_{\gamma \neq \alpha,-\beta} \mathbf{C}(\gamma, \alpha-\beta) \widetilde{S}_{\alpha-\gamma}^{k} \widetilde{S}_{\beta+\gamma}^{k} \tilde{\mathbf{f}}_{\alpha, \beta, \gamma}^{\eta}+ \\
& +\mathbf{C}(\alpha, \beta) \widetilde{S}_{0}^{k} \widetilde{S}_{\alpha+\beta}^{k}\left(E_{1}(\breve{\alpha}+\breve{\beta}+\eta)-E_{1}(\breve{\alpha})-E_{1}(\breve{\beta})-E_{1}(\eta)\right)- \\
& -\mathbf{C}(\alpha, \beta) \sum_{j \neq k}\left[\widetilde{S}_{0}^{k} \widetilde{S}_{\alpha+\beta}^{j} \varphi_{\alpha+\beta, \eta}\left(x_{k}-x_{j}\right)-\widetilde{S}_{0}^{j} \widetilde{S}_{\alpha+\beta}^{k} \varphi_{\eta}\left(x_{k}-x_{j}\right)\right]- \\
& -2 \sum_{j \neq k} \mathbf{C}(\gamma, \alpha-\beta) \widetilde{S}_{\alpha-\gamma}^{k} \widetilde{S}_{\beta+\gamma}^{k} \varphi_{\beta+\gamma, \eta}\left(x_{k}-x_{j}\right),
\end{aligned}
$$

где

$$
\begin{aligned}
\tilde{\mathbf{f}}_{\alpha, \beta, \gamma}^{\eta} & =E_{1}(\breve{\gamma})-E_{1}(\breve{\alpha}-\breve{\beta}-\breve{\gamma})+E_{1}(\breve{\alpha}-\breve{\gamma}+\eta)-E_{1}(\breve{\beta}+\breve{\gamma}+\eta), \\
\left\{\widetilde{S}_{\alpha}^{j}, \widetilde{S}_{\beta}^{k}\right\} & =\sum_{\gamma \neq \alpha,-\beta} \mathbf{C}(\gamma, \alpha-\beta) \widetilde{S}_{\alpha-\gamma}^{j} \widetilde{S}_{\beta+\gamma}^{k} \varphi_{\gamma, \eta}\left(x_{j}-x_{k}\right)-
\end{aligned}
$$




$$
-\mathbf{C}(\alpha, \beta)\left(\widetilde{S}_{0}^{j} \widetilde{S}_{\alpha+\beta}^{k} \varphi_{\alpha, \eta}\left(x_{j}-x_{k}\right)-\widetilde{S}_{0}^{k} \widetilde{S}_{\alpha+\beta}^{j} \varphi_{-\beta, \eta}\left(x_{k}-x_{j}\right)\right) .
$$

Докажем эквивалентность, положив $\tilde{x}_{i}=0$ для некоторого $i$.

ПредЛОЖЕНИЕ 4. Забиксируем два индекса $1 \leqslant i, k \leqslant n, i \neq k$. Определим

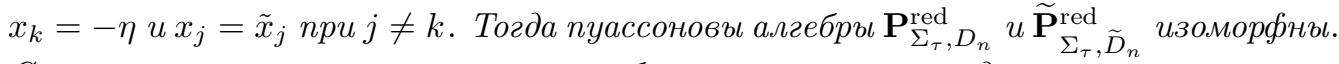
Соответствующие канонические преобразования имеют вид

$$
\begin{gathered}
S_{0}=\widetilde{S}_{0}^{i}+\sum_{j \neq i}^{n} \frac{1}{\varphi_{\eta}\left(\tilde{x}_{j}\right)}\left(E_{1}\left(\tilde{x}_{j}\right)+E_{1}(\eta)\right) \widetilde{S}_{0}^{j}, \\
S_{0}^{k}=-\sum_{j \neq i}^{n} \frac{\widetilde{S}_{0}^{j}}{\varphi_{\eta}\left(\tilde{x}_{j}\right)}, \quad S_{\alpha}^{k}=\frac{\widetilde{S}_{\alpha}^{i}}{\varphi_{\alpha}(\eta)}+\sum_{j \neq i} \frac{\varphi_{-\alpha}\left(\tilde{x}_{j}\right) \widetilde{S}_{\alpha}^{j}}{\varphi_{\eta}\left(\tilde{x}_{j}\right) \varphi_{\alpha}(\eta)}, \\
S_{0}^{j \neq k}=\frac{\widetilde{S}_{0}^{j}}{\varphi_{\eta}\left(\tilde{x}_{j}\right)}, \quad S_{\alpha}^{j \neq k}=\frac{\widetilde{S}_{\alpha}^{j}}{\varphi_{\eta}\left(\tilde{x}_{j}\right)} .
\end{gathered}
$$

ДокАзАтельство. Оператор Лакса $L_{\widetilde{D}_{n}}^{\eta}(3.7)$ после деления на $\varphi_{\eta}(z)$ приобретает те же монодромии, что и матрица $L(2.21)$. Рассмотрим вычеты и постоянные члены этих операторов. Имеем

$$
\frac{L_{\widetilde{D}_{n}}^{\eta}}{\varphi_{\eta}(z)}=\widetilde{S}_{0}^{i} T_{0}+\sum_{j \neq i}^{n}\left[\widetilde{S}_{0}^{j} \frac{\varphi_{\eta}\left(z-\tilde{x}_{j}\right)}{\varphi_{\eta}(z)} T_{0}+\sum_{\alpha}\left(\widetilde{S}_{\alpha}^{j} \frac{\varphi_{\alpha, \eta}\left(z-\tilde{x}_{j}\right)}{\varphi_{\eta}(z)}+\widetilde{S}_{\alpha}^{i} \frac{\varphi_{\alpha, \eta}(z)}{\varphi_{\eta}(z)}\right) T_{\alpha}\right] .
$$

Применяя соотношения (Б.9), (Б.10) и (Б.11), мы получаем

$$
\begin{aligned}
& \frac{L_{\widetilde{D}_{n}}^{\eta}}{\varphi_{\eta}(z)}=\left(\widetilde{S}_{0}^{i}+\sum_{j \neq i}^{n} \frac{1}{\varphi_{\eta}\left(\tilde{x}_{j}\right)}\left(E_{1}\left(\tilde{x}_{j}\right)+E_{1}(\eta)\right) \widetilde{S}_{0}^{j}\right) T_{0}- \\
&-E_{1}(z+\eta) \sum_{j \neq i}^{n} \frac{\widetilde{S}_{0}^{j}}{\varphi_{\eta}\left(\tilde{x}_{j}\right)} \cdot T_{0}+\sum_{j \neq i}^{n} E_{1}\left(z-\tilde{x}_{j}\right) \cdot \frac{\widetilde{S}_{0}^{j}}{\varphi_{\eta}\left(\tilde{x}_{j}\right)} T_{0}+ \\
&+\sum_{\alpha, j \neq i} \varphi_{\alpha}\left(z-\tilde{x}_{j}\right) \frac{\widetilde{S}_{\alpha}^{j}}{\varphi_{\eta}\left(\tilde{x}_{j}\right)} \cdot T_{\alpha}+ \\
&+\sum_{\alpha, j \neq i} \varphi_{\alpha}(z+\eta) \cdot\left(\frac{\varphi_{-\alpha}\left(\tilde{x}_{j}\right) \widetilde{S}_{\alpha}^{j}}{\varphi_{\eta}\left(\tilde{x}_{j}\right) \varphi_{\alpha}(\eta)}+\frac{\widetilde{S}_{\alpha}^{i}}{\varphi_{\alpha}(\eta)}\right) T_{\alpha} .
\end{aligned}
$$

Заметим, что в этом случае возникает новый полюс в точке $x_{b}=-\eta$. Сравнивая с (2.21), мы приходим к уравнению (3.12).

3.3. Бигамильтонова структура. Введем в пространстве $\mathbb{C}^{n N^{2}}$ линейные скобки Ли-Пуассона. Для этого рассмотрим $n$ копий $g l(N, \mathbb{C}): \mathfrak{g}^{*}=g l(N, \mathbb{C}) \oplus \cdots \oplus$ $g l(N, \mathbb{C})$ со скобками $\left.{ }^{2}\right)$

$$
\left\{S_{\alpha}^{j}, S_{\beta}^{k}\right\}_{1}=C(\alpha, \beta) S_{\alpha+\beta}^{j} \delta^{j k}
$$

\footnotetext{
${ }^{2)}$ Индекс 1 у скобок $\{\cdot, \cdot\}_{1}$ означает линейные скобки.
} 
ЗАмЕчАНИЕ 3. Скобки Ли-Пуассона имеют $r$-матричную форму

$$
\left\{\widetilde{L}_{1}(z), \widetilde{L}_{2}(w)\right\}=\left[r(z-w), \widetilde{L}_{1}(z)+\widetilde{L}_{2}(w)\right]
$$

где $r$-матрица та же, что и для квадратичных скобок $(2.14), \widetilde{L}=\sum_{j=1}^{n} \widetilde{L}_{j}(z)(2.21)$.

Две пуассоновы структуры называются совместными (образуют пуассонову па$p y)$, если их линейные комбинации также являются пуассоновыми структурами.

ПРЕДЛОЖЕНИЕ 5. Линейные (3.14) и квадратичные (3.2)-(3.6) скобки Пуассона на пространстве $\mathbb{C}^{n N^{2}}$ совместны.

ДокАЗАТЕЛЬство. Выберем точку $x_{k} \in \widetilde{D}_{n}$ и заменим $\widetilde{S}_{0}^{k}$ на $\widetilde{S}_{0}^{k}+\lambda$, где $\lambda \in$ $\mathbb{C}$ - число. Поэтому $\widetilde{S}_{0}^{k}+\lambda$ коммутирует относительно скобки Пуассона со всеми элементами квадратичной пуассоновой алгебры. Подставим эту новую переменную в (3.9) и (3.11). Замена переменных не нарушает тождества Якоби, и, таким образом, мы приходим к следующей пуассоновой структуре:

$$
\{\widetilde{S}, \widetilde{S}\}_{\lambda}:=\{\widetilde{S}, \widetilde{S}\}_{2}+\lambda\{\widetilde{S}, \widetilde{S}\}_{1}
$$

Рассмотрим члены, относящиеся к линейным скобкам,

$$
\begin{array}{ll}
\left\{\widetilde{S}_{\alpha}^{k}, \widetilde{S}_{\beta}^{k}\right\}_{1}=F_{1} \widetilde{S}_{\alpha+\beta}^{j}+F_{2} \widetilde{S}_{\alpha+\beta}^{k}, & \left\{\widetilde{S}_{\alpha}^{k}, \widetilde{S}_{\beta}^{j}\right\}_{1}=G_{2} \widetilde{S}_{\alpha+\beta}^{j}, \\
\left\{\widetilde{S}_{\alpha}^{j}, \widetilde{S}_{\beta}^{k}\right\}_{1}=\widetilde{G}_{2} \widetilde{S}_{\alpha+\beta}^{j}, & \left\{\widetilde{S}_{\alpha}^{j}, \widetilde{S}_{\beta}^{j}\right\}_{1}=H_{2} \widetilde{S}_{\alpha+\beta}^{j},
\end{array}
$$

где, с точностью до общего множителя $C(\alpha, \beta)$, коэффициенты имеют форму

$$
\begin{gathered}
F_{1}=\varphi_{\alpha+\beta, \eta}\left(x_{k j}\right), \quad F_{2}=-E_{1}(\breve{\alpha})-E_{1}(\breve{\beta})-E_{1}(\eta)+E_{1}(\breve{\alpha}+\breve{\beta}+\eta), \\
G_{2}=-\varphi_{\alpha}\left(x_{k j}\right), \quad \widetilde{G}_{2}=-\varphi_{\beta}\left(x_{k j}\right), \quad H_{2}=\varphi_{0,-\eta}\left(x_{k j}\right),
\end{gathered}
$$

$x_{k j}=x_{k}-x_{j}$. Следующая лемма завершает доказательство предложения.

ЛЕмма. Линейная пуассонова алгебра (3.15) эквивалентна прямой сумме алгебр Ли-Пуассона на $\bigoplus_{l=1}^{n} g l(N, \mathbb{C})$ :

$$
\left\{t_{\alpha}^{j}, t_{\beta}^{k}\right\}=C(\alpha, \beta) t_{\alpha+\beta}^{j} \delta^{j k} .
$$

ДокАЗАТЕЛЬСтво. Определим

$$
\widetilde{S}_{\alpha}^{k}=a_{\alpha} t_{\alpha}^{k}+b_{\alpha} t_{\alpha}^{j}, \quad \widetilde{S}_{\alpha}^{j}=H_{2} t_{\alpha}^{j} .
$$

Скобки (3.15) эквивалентны (3.17) при

$$
a_{\alpha} a_{\beta}=a_{\alpha+\beta} F_{2}, \quad b_{\alpha}=G_{2}, \quad b_{\alpha} b_{\beta}=F_{1} H_{2}+b_{\alpha+\beta} F_{2} .
$$

Решим эти уравнения. Решение первого уравнения, которое может быть найдено из (А.10), имеет следующий вид: $a_{\alpha}=-\varphi_{\alpha}(\eta)$. Далее докажем, что $b_{\alpha}=G_{2}=$ $-\varphi_{\alpha}\left(x_{k j}\right)$ удовлетворяет последнему соотношению в (3.18). При $b_{\alpha}=-\varphi_{\alpha}\left(x_{k j}\right)$ оно будет иметь следующий вид:

$$
\varphi_{\alpha}\left(x_{k j}\right) \varphi_{\beta}\left(x_{k j}\right)=\varphi_{\alpha+\beta, \eta}\left(x_{k j}\right) \varphi_{0,-\eta}\left(x_{k j}\right)+
$$




$$
+\varphi_{\alpha+\beta}\left(x_{k j}\right)\left(E_{1}(\breve{\alpha})+E_{1}(\breve{\beta})+E_{1}(\eta)-E_{1}(\breve{\alpha}+\breve{\beta}+\eta)\right) .
$$

Из соотношения (А.9) следует, что

$\varphi_{\alpha+\beta, \eta}\left(x_{k j}\right) \varphi_{0,-\eta}\left(x_{k j}\right)=\varphi_{\alpha+\beta}\left(x_{k j}\right)\left(E_{1}(\breve{\alpha}+\breve{\beta}+\eta)+E_{1}(-\eta)+E_{1}\left(x_{k j}\right)-E_{1}\left(\breve{\alpha}+\breve{\beta}+x_{k j}\right)\right)$, поэтому последнее соотношение в (3.18) является тождеством. Таким образом, мы приходим к (3.17).

\section{4. КВАНТОВАЯ АЛГЕБРА}

4.1. Общий случай. В этом разделе мы рассматриваем квантование квадратичной пуассоновой алгебры для случая $n>1$. Пусть квантовая $R$-матрица имеет следующий вид:

$$
R(z, w)=\sum_{a \in \mathbb{Z}_{N}^{(2)}} \varphi_{a}^{\hbar}(z-w) T_{a} \otimes T_{-a}
$$

где $\varphi_{a}^{\hbar}(z) \equiv \varphi_{\hbar, a}(z)$. Заметим, что в отличие от классической $r$-матрицы здесь появляется добавочный член $\varphi_{0}^{\hbar}(z-w) \sigma_{0} \otimes \sigma_{0}$. Квантовая $R$-матрица удовлетворяет квантовому уравнению Янга-Бакстера

$$
R_{12}(z-w) R_{13}(z) R_{23}(w)=R_{23}(w) R_{13}(z) R_{12}(z-w) .
$$

С помощью квантового уравнения Янга-Бакстера ассоциативная алгебра определяется следующим образом:

$$
R(z-w) L_{1}^{\hbar}(z) L_{2}^{\hbar}(w)=L_{2}^{\hbar}(w) L_{1}^{\hbar}(z) R(z-w) .
$$

Оператор Лакса в (4.3) имеет следующие монодромии относительно $\hbar$ :

$$
L^{\hbar+\tau}(z)=\mathbf{e}_{N}(-z) L^{\hbar}(z), \quad L^{\hbar+1}(z)=L^{\hbar}(z) .
$$

Поэтому мы должны предположить, что переменные $S$ зависят от $\hbar$ и $x_{j}$. Новые переменные и оператор Лакса в (4.3) будут иметь вид

$$
\begin{gathered}
S_{\text {new }}^{j}=\widehat{S}_{0}^{j} \varphi_{0}^{\hbar}\left(x_{j}\right), \\
L^{\hbar}(z)=\sum_{j=1}^{n}\left(\widehat{S}_{0}^{j} \varphi_{0}^{\hbar}\left(x_{j}\right) \varphi_{0}^{\hbar}\left(z-x_{j}\right) T_{0}+\sum_{\alpha} \widehat{S}_{\alpha}^{j} \varphi_{\alpha}^{\hbar}\left(x_{j}\right) \varphi_{\alpha}^{\hbar}\left(z-x_{j}\right) T_{\alpha}\right)= \\
=\sum_{j=1}^{n} \sum_{a \in \mathbb{Z}_{N}^{(2)}} \widehat{S}_{a}^{j} \varphi_{a}^{\hbar}\left(x_{j}\right) \varphi_{a}^{\hbar}\left(z-x_{j}\right) T_{a} .
\end{gathered}
$$

ПРЕДЛОЖЕНИЕ 6. Соотношения в ассоциативной алгебре принимают следующий вид:

$$
\sum_{c} f^{\hbar}(a, b, c) \cdot \widehat{S}_{b+c}^{j} \widehat{S}_{a-c}^{j} \varphi_{b+c}^{\hbar}\left(x_{j}\right) \varphi_{a-c}^{\hbar}\left(x_{j}\right) \mathbf{e}_{N}\left(\frac{c \times(a-b)}{2}\right)+
$$




$$
\begin{aligned}
&+\sum_{c} \sum_{k \neq j} \varphi_{a-c}^{\hbar}\left(x_{j}-x_{k}\right) \varphi_{b+c}^{\hbar}\left(x_{j}\right) \varphi_{a-c}^{\hbar}\left(x_{k}\right) \times \\
& \times\left(\widehat{S}_{b+c}^{j} \widehat{S}_{a-c}^{k} \mathbf{e}_{N}\left(\frac{c \times(a-b)}{2}\right)-\widehat{S}_{a-c}^{k} \widehat{S}_{b+c}^{j} \mathbf{e}_{N}\left(\frac{c \times(a-b)}{2}\right)\right)=0 \\
& \sum_{c} \varphi_{c}^{\hbar}\left(x_{j}-x_{k}\right) \varphi_{b+c}^{\hbar}\left(x_{k}\right) \varphi_{a-c}^{\hbar}\left(x_{j}\right) \times \\
& \quad \times\left(\widehat{S}_{a-c}^{j} \widehat{S}_{b+c}^{k} \mathbf{e}_{N}\left(-\frac{c \times(a-b)}{2}\right)-\widehat{S}_{b+c}^{k} \widehat{S}_{a-c}^{j} \mathbf{e}_{N}\left(\frac{c \times(a-b)}{2}\right)\right)=0
\end{aligned}
$$

əде $k \neq j$

$$
f^{\hbar}(a, b, c)=E_{1}(c+\hbar)-E_{1}(a-b-c+\hbar)+E_{1}(a-c+\hbar)-E_{1}(b+c+\hbar),
$$

$a, b, c \in \mathbb{Z}_{N}^{(2)}$

ДоказАтельство. Рассмотрим некоторый матричный элемент $T_{a} \otimes T_{b}$. Подставляя (4.5) и (4.1) в (4.3), получаем следующие выражения:

$$
\begin{aligned}
& \sum_{j, k} \sum_{c, a, b} \varphi_{c}^{\hbar}(z-w) \varphi_{a}^{\hbar}\left(z-x_{j}\right) \varphi_{b}^{\hbar}\left(w-x_{k}\right) \cdot \widehat{S}_{a}^{j} \widehat{S}_{b}^{k} \varphi_{b}^{\hbar}\left(x_{k}\right) \varphi_{a}^{\hbar}\left(x_{j}\right) \cdot T_{c} T_{a} \otimes T_{-c} T_{b}= \\
& =\sum_{j, k} \sum_{c, a, b} \varphi_{c}^{\hbar}(z-w) \varphi_{a}^{\hbar}\left(z-x_{j}\right) \varphi_{b}^{\hbar}\left(w-x_{k}\right) \times \\
& \quad \times \widehat{S}_{b}^{k} \widehat{S}_{a}^{j} \varphi_{b}^{\hbar}\left(x_{k}\right) \varphi_{a}^{\hbar}\left(x_{j}\right) \cdot T_{a} T_{c} \otimes T_{b} T_{-c} . \\
& \sum_{j, k} \sum_{c, a, b} \varphi_{c}^{\hbar}(z-w) \varphi_{a}^{\hbar}\left(z-x_{j}\right) \varphi_{b}^{\hbar}\left(w-x_{k}\right) \times \\
& \quad \times \widehat{S}_{a}^{j} \widehat{S}_{b}^{k} \varphi_{b}^{\hbar}\left(x_{k}\right) \varphi_{a}^{\hbar}\left(x_{j}\right) \mathbf{e}_{N}\left(-\frac{c \times(a-b)}{2}\right) \cdot T_{c+a} \otimes T_{-c+b}= \\
& =\sum_{j, k} \sum_{c, a, b} \varphi_{c}^{\hbar}(z-w) \varphi_{a}^{\hbar}\left(z-x_{j}\right) \varphi_{b}^{\hbar}\left(w-x_{k}\right) \times \\
& \quad \times \widehat{S}_{b}^{k} \widehat{S}_{a}^{j} \varphi_{b}^{\hbar}\left(x_{k}\right) \varphi_{a}^{\hbar}\left(x_{j}\right) \mathbf{e}_{N}\left(\frac{c \times(a-b)}{2}\right) \cdot T_{a+c} \otimes T_{b-c} .
\end{aligned}
$$

Функции в левой и в правой частях выражений эквивалентны, так как их полюсы и квазипериоды совпадают. После замены переменных $a \rightarrow a-c, b \rightarrow b+c$ коэффициенты при матричном элементе $T_{a} \otimes T_{b}$ принимают вид

$$
\begin{aligned}
& \sum_{c} \varphi_{c}^{\hbar}(z-w) \varphi_{a-c}^{\hbar}\left(z-x_{j}\right) \varphi_{b+c}^{\hbar}\left(w-x_{k}\right) \varphi_{b+c}^{\hbar}\left(x_{k}\right) \varphi_{a-c}^{\hbar}\left(x_{j}\right) \times \\
& \quad \times\left(\widehat{S}_{a-c}^{j} \widehat{S}_{b+c}^{k} \mathbf{e}_{N}\left(-\frac{c \times(a-b)}{2}\right)-\widehat{S}_{b+c}^{k} \widehat{S}_{a-c}^{j} \mathbf{e}_{N}\left(\frac{c \times(a-b)}{2}\right)\right)=0 .
\end{aligned}
$$


Рассмотрим два типа этих выражений:

$$
\begin{aligned}
\sum_{c} \varphi_{c}^{\hbar}(z-w) \varphi_{a-c}^{\hbar}\left(z-x_{j}\right) \varphi_{b+c}^{\hbar}\left(w-x_{k}\right) \varphi_{b+c}^{\hbar}\left(x_{k}\right) \varphi_{a-c}^{\hbar}\left(x_{j}\right) \times \\
\quad \times\left(\widehat{S}_{a-c}^{j} \widehat{S}_{b+c}^{k} \mathbf{e}_{N}\left(-\frac{c \times(a-b)}{2}\right)-\right. \\
\left.\quad-\widehat{S}_{b+c}^{k} \widehat{S}_{a-c}^{j} \mathbf{e}_{N}\left(\frac{c \times(a-b)}{2}\right)\right)=0, \quad k \neq j, \\
\sum_{c}\left(\varphi_{c}^{\hbar}(z-w) \varphi_{a-c}^{\hbar}\left(z-x_{j}\right) \varphi_{b+c}^{\hbar}\left(w-x_{j}\right)-\right. \\
\left.\quad-\varphi_{a-b-c}^{\hbar}(z-w) \varphi_{a-c}^{\hbar}\left(z-x_{j}\right) \varphi_{b+c}^{\hbar}\left(w-x_{k}\right)\right) \times \\
\quad \times \widehat{S}_{a-c}^{j} \widehat{S}_{b+c}^{k} \varphi_{b+c}^{\hbar}\left(x_{k}\right) \varphi_{a-c}^{\hbar}\left(x_{j}\right) \mathbf{e}_{N}\left(-\frac{c \times(a-b)}{2}\right)=0, \quad k=j .
\end{aligned}
$$

Второе выражение получаем после замены $c \rightarrow a-b-c$. В пределе при $z \rightarrow x_{j}$, $w \rightarrow x_{j}$ и $z \rightarrow x_{j}, w \rightarrow x_{k}$, как и в разделе 3 , мы получаем коэффициенты, которые равны нулю. Таким образом, предложение доказано.

4.2. Квадратичная алгебра в случае $G L(2, \mathbb{C})$. Рассмотрим случай $N=2$ более подробно. В этом случае квантовая $R$-матрица имеет вид

$$
R(z, w)=\sum_{a=0}^{3} \varphi_{a}^{\hbar}(z-w) \sigma_{a} \otimes \sigma_{a}
$$

где вместо $T_{a}$ мы используем базис сигма-матриц.

ПРЕДЛОЖЕНИЕ 7. Соотношения в ассоииативной алгебре принимают следующий вид: при $k=j$

$$
\begin{aligned}
& {\left[\widehat{S}_{\alpha}^{j}, \widehat{S}_{\beta}^{j}\right]_{-}=} i \varepsilon_{\alpha \beta \gamma} c_{1}^{1}(j, j ; \alpha, \beta, \gamma)\left[\widehat{S}_{\gamma}^{j}, \widehat{S}_{0}^{j}\right]_{+}+ \\
&+\sum_{k \neq j} i \varepsilon_{\alpha \beta \gamma} \frac{1}{k_{\alpha}}\left(\varphi_{\gamma}^{\hbar}\left(x_{j k}\right) c_{1}^{2}(j, k ; \alpha, \beta, \gamma)\left[\widehat{S}_{\gamma}^{k}, \widehat{S}_{0}^{j}\right]_{+}-\right. \\
&\left.-\varphi_{0}^{\hbar}\left(x_{j k}\right) c_{1}^{3}(j, k ; \alpha, \beta, \gamma)\left[\widehat{S}_{\gamma}^{j}, \widehat{S}_{0}^{k}\right]_{+}\right), \\
& c_{1}^{1}(j, j ; \alpha, \beta, \gamma)= \frac{\varphi_{\gamma}^{\hbar}\left(x_{j}\right) \varphi_{0}^{\hbar}\left(x_{j}\right)}{\varphi_{\alpha}^{\hbar}\left(x_{j}\right) \varphi_{\beta}^{\hbar}\left(x_{j}\right)}, \quad c_{1}^{2}(j, k ; \alpha, \beta, \gamma)=\frac{\varphi_{\gamma}^{\hbar}\left(x_{k}\right) \varphi_{0}^{\hbar}\left(x_{j}\right)}{\varphi_{\alpha}^{\hbar}\left(x_{j}\right) \varphi_{\beta}^{\hbar}\left(x_{j}\right)}, \\
& c_{1}^{3}(j, k ; \alpha, \beta, \gamma)=\frac{\varphi_{\gamma}^{\hbar}\left(x_{j}\right) \varphi_{0}^{\hbar}\left(x_{k}\right)}{\varphi_{\alpha}^{\hbar}\left(x_{j}\right) \varphi_{\beta}^{\hbar}\left(x_{j}\right)}, \\
& {\left[\widehat{S}_{\alpha}^{j}, \widehat{S}_{0}^{j}\right]_{-}=i \varepsilon_{\alpha \beta \gamma} \frac{J_{\beta}-J_{\gamma}}{J_{\alpha}} c_{2}^{1}(j, j ; \alpha, \beta, \gamma)\left[\widehat{S}_{\beta}^{j}, \widehat{S}_{\gamma}^{j}\right]_{+}+} \\
&+\sum_{k \neq j} i \varepsilon_{\alpha \beta \gamma} \frac{1}{k_{\alpha} J_{\alpha}}\left(c_{2}^{2}(j, k ; \alpha, \beta, \gamma) D(\alpha, \beta)\left[\widehat{S}_{\gamma}^{j}, \widehat{S}_{\beta}^{k}\right]_{+}-\right. \\
&-\left.c_{2}^{3}(j, k ; \alpha, \beta, \gamma) D(\alpha, \gamma)\left[\widehat{S}_{\gamma}^{k}, \widehat{S}_{\beta}^{j}\right]_{+}\right),
\end{aligned}
$$




$$
\begin{gathered}
c_{2}^{1}(j, j ; \alpha, \beta, \gamma)=\frac{\varphi_{\gamma}^{\hbar}\left(x_{j}\right) \varphi_{\beta}^{\hbar}\left(x_{j}\right)}{\varphi_{\alpha}^{\hbar}\left(x_{j}\right) \varphi_{0}^{\hbar}\left(x_{j}\right)}, \quad c_{2}^{2}(j, k ; \alpha, \beta, \gamma)=\frac{\varphi_{\gamma}^{\hbar}\left(x_{j}\right) \varphi_{\beta}^{\hbar}\left(x_{k}\right)}{\varphi_{\alpha}^{\hbar}\left(x_{j}\right) \varphi_{0}^{\hbar}\left(x_{j}\right)}, \\
c_{2}^{3}(j, k ; \alpha, \beta, \gamma)=\frac{\varphi_{\gamma}^{\hbar}\left(x_{k}\right) \varphi_{\beta}^{\hbar}\left(x_{j}\right)}{\varphi_{\alpha}^{\hbar}\left(x_{j}\right) \varphi_{0}^{\hbar}\left(x_{j}\right)}, \\
D(\alpha, \beta)=\left(k_{\alpha}\left(k_{\alpha}-k_{\beta}-\ln ^{\prime} \varphi_{\alpha}^{\hbar}\left(x_{j k}\right)\right)+\ln ^{\prime} \frac{\varphi_{\alpha}^{\hbar}\left(x_{j k}\right)}{\varphi_{0}^{\hbar}\left(x_{j k}\right)}\left(\partial_{z}-E_{1}(\hbar)\right)\right) \varphi_{\beta}^{\hbar}\left(x_{j k}\right), \\
\ln ^{\prime} \varphi_{u}^{\hbar}(x)=\frac{\partial_{u}\left(\varphi_{u}^{\hbar}(x)\right)}{\varphi_{u}^{\hbar}(x)},
\end{gathered}
$$

npu $k \neq j:$

$$
\begin{gathered}
{\left[\widehat{S}_{\alpha}^{j}, \widehat{S}_{\beta}^{k}\right]_{-}=i \varepsilon_{\alpha \beta \gamma} \frac{1}{k_{\gamma}}\left(\varphi_{\beta}\left(x_{j k}\right) c_{3}^{1}(j, k ; \alpha, \beta, \gamma)\left[\widehat{S}_{\gamma}^{j}, \widehat{S}_{0}^{k}\right]_{+}-\right.} \\
\left.-\varphi_{\alpha}\left(x_{j k}\right) c_{3}^{2}(j, k ; \alpha, \beta, \gamma)\left[\widehat{S}_{\gamma}^{k}, \widehat{S}_{0}^{j}\right]_{+}\right) \\
c_{3}^{1}(j, k ; \alpha, \beta, \gamma)=\frac{\varphi_{\gamma}^{\hbar}\left(x_{j}\right) \varphi_{0}^{\hbar}\left(x_{k}\right)}{\varphi_{\alpha}^{\hbar}\left(x_{j}\right) \varphi_{\beta}^{\hbar}\left(x_{k}\right)}, \quad c_{3}^{2}(j, k ; \alpha, \beta, \gamma)=\frac{\varphi_{\gamma}^{\hbar}\left(x_{k}\right) \varphi_{0}^{\hbar}\left(x_{j}\right)}{\varphi_{\alpha}^{\hbar}\left(x_{j}\right) \varphi_{\beta}^{\hbar}\left(x_{k}\right)}, \\
{\left[\widehat{S}_{\alpha}^{j}, \widehat{S}_{0}^{k}\right]_{-}=i \varepsilon_{\alpha \beta \gamma} \frac{1}{k_{\alpha}}\left(\varphi_{\beta}\left(x_{j k}\right) c_{4}^{1}(j, k ; \alpha, \beta, \gamma)\left[\widehat{S}_{\gamma}^{j}, \widehat{S}_{\beta}^{k}\right]_{+}-\right.} \\
\left.-\varphi_{\alpha}\left(x_{j k}\right) c_{4}^{2}(j, k ; \alpha, \beta, \gamma)\left[\widehat{S}_{\gamma}^{k}, \widehat{S}_{\beta}^{j}\right]_{+}\right) \\
c_{4}^{1}(j, k ; \alpha, \beta, \gamma)=\frac{\varphi_{\gamma}^{\hbar}\left(x_{j}\right) \varphi_{\beta}^{\hbar}\left(x_{k}\right)}{\varphi_{\alpha}^{\hbar}\left(x_{j}\right) \varphi_{0}^{\hbar}\left(x_{k}\right)}, \quad c_{4}^{2}(j, k ; \alpha, \beta, \gamma)=\frac{\varphi_{\gamma}^{\hbar}\left(x_{k}\right) \varphi_{\beta}^{\hbar}\left(x_{j}\right)}{\varphi_{\alpha}^{\hbar}\left(x_{j}\right) \varphi_{0}^{\hbar}\left(x_{k}\right)}
\end{gathered}
$$

где $k_{\gamma}=E_{1}(\breve{\gamma}+\hbar)-E_{1}(\breve{\gamma})-E_{1}(\hbar), J_{\gamma}=E_{2}(\breve{\gamma}+\hbar)-E_{2}(\hbar), x_{j k}=x_{j}-x_{k}$.

ДокАЗАтЕльство. Подставим (4.5) в (4.3) при $N=2$ и проверим "баланс" коэффициентов двух типов матричных элементов $\sigma_{\alpha} \otimes \sigma_{\beta}$ и $\sigma_{\alpha} \otimes \sigma_{0}$ левой и правой частей равенства. Зафиксируем эти элементы и сравним коэффициенты в соответствующих полюсах. Получим следующие выражения для скобок:

$$
\begin{aligned}
{\left[\widehat{S}_{\alpha}^{j}, \widehat{S}_{\beta}^{j}\right]_{-}=i \varepsilon_{\alpha \beta \gamma} \frac{\varphi_{\gamma}^{\hbar}\left(x_{j}\right) \varphi^{\hbar}\left(x_{j}\right)}{\varphi_{\alpha}^{\hbar}\left(x_{j}\right) \varphi_{\beta}^{\hbar}\left(x_{j}\right)}\left[\widehat{S}_{\gamma}^{j}, \widehat{S}_{0}^{j}\right]_{+}+\sum_{k \neq j} \frac{\varphi_{\gamma}^{\hbar}\left(x_{j k}\right)}{f^{\hbar}(\alpha, \beta, 0)} \cdot \frac{\varphi_{\alpha}^{\hbar}\left(x_{j}\right) \varphi_{\beta}^{\hbar}\left(x_{k}\right)}{\varphi_{\alpha}^{\hbar}\left(x_{j}\right) \varphi_{\beta}^{\hbar}\left(x_{j}\right)}\left[\widehat{S}_{\alpha}^{j}, \widehat{S}_{\beta}^{k}\right]_{-}-} \\
\quad-\frac{\varphi_{\alpha}^{\hbar}\left(x_{j k}\right)}{f^{\hbar}(\alpha, \beta, 0)} \cdot \frac{\varphi_{\alpha}^{\hbar}\left(x_{k}\right) \varphi_{\beta}^{\hbar}\left(x_{j}\right)}{\varphi_{\alpha}^{\hbar}\left(x_{j}\right) \varphi_{\beta}^{\hbar}\left(x_{j}\right)}\left[\widehat{S}_{\alpha}^{k}, \widehat{S}_{\beta}^{j}\right]_{-}+ \\
+i \varepsilon_{\alpha \beta \gamma} \frac{\varphi_{0}^{\hbar}\left(x_{j k}\right)}{f^{\hbar}(\alpha, \beta, 0)} \cdot \frac{\varphi_{\gamma}^{\hbar}\left(x_{k}\right) \varphi_{0}^{\hbar}\left(x_{j}\right)}{\varphi_{\alpha}^{\hbar}\left(x_{j}\right) \varphi_{\beta}^{\hbar}\left(x_{j}\right)}\left[\widehat{S}_{\gamma}^{j}, \widehat{S}_{0}^{k}\right]_{+}- \\
-i \varepsilon_{\alpha \beta \gamma} \frac{\varphi_{\gamma}^{\hbar}\left(x_{j k}\right)}{f^{\hbar}(\alpha, \beta, 0)} \cdot \frac{\varphi_{\gamma}^{\hbar}\left(x_{j}\right) \varphi_{0}^{\hbar}\left(x_{k}\right)}{\varphi_{\alpha}^{\hbar}\left(x_{j}\right) \varphi_{\beta}^{\hbar}\left(x_{j}\right)}\left[\widehat{S}_{\gamma}^{k}, \widehat{S}_{0}^{j}\right]_{+},
\end{aligned}
$$

$\left[\widehat{S}_{\alpha}^{j}, \widehat{S}_{0}^{j}\right]_{-}=i \varepsilon_{\alpha \beta \gamma} \frac{J_{\beta}-J_{\gamma}}{J_{\alpha}} \cdot \frac{\varphi_{\gamma}^{\hbar}\left(x_{j}\right) \varphi_{\beta}^{\hbar}\left(x_{j}\right)}{\varphi_{\alpha}^{\hbar}\left(x_{j}\right) \varphi_{0}^{\hbar}\left(x_{j}\right)}\left[\widehat{S}_{\beta}^{j}, \widehat{S}_{\gamma}^{j}\right]_{+}+$

$$
+\sum_{k \neq j} \frac{\varphi_{0}^{\hbar}\left(x_{j k}\right)}{J_{\alpha}} \cdot \frac{\varphi_{0}^{\hbar}\left(x_{k}\right)}{\varphi_{0}^{\hbar}\left(x_{j}\right)}\left[\widehat{S}_{\alpha}^{j}, \widehat{S}_{0}^{k}\right]_{-}+\frac{\varphi_{\alpha}^{\hbar}\left(x_{j k}\right)}{J_{\alpha}} \cdot \frac{\varphi_{\alpha}^{\hbar}\left(x_{k}\right)}{\varphi_{\alpha}^{\hbar}\left(x_{j}\right)}\left[\widehat{S}_{\alpha}^{k}, \widehat{S}_{0}^{j}\right]_{-}+
$$




$$
\begin{aligned}
& +i \varepsilon_{\alpha \beta \gamma} \frac{\varphi_{\gamma}^{\hbar}\left(x_{j k}\right)}{J_{\alpha}} \cdot \frac{\varphi_{\gamma}^{\hbar}\left(x_{k}\right) \varphi_{\beta}^{\hbar}\left(x_{j}\right)}{\varphi_{\alpha}^{\hbar}\left(x_{j}\right) \varphi_{0}^{\hbar}\left(x_{j}\right)}\left[\widehat{S}_{\beta}^{j}, \widehat{S}_{\gamma}^{k}\right]_{+}+ \\
& +i \varepsilon_{\alpha \beta \gamma} \frac{\varphi_{\beta}^{\hbar}\left(x_{j k}\right)}{J_{\alpha}} \cdot \frac{\varphi_{\gamma}^{\hbar}\left(x_{j}\right) \varphi_{\beta}^{\hbar}\left(x_{k}\right)}{\varphi_{\alpha}^{\hbar}\left(x_{j}\right) \varphi_{0}^{\hbar}\left(x_{j}\right)}\left[\widehat{S}_{\gamma}^{j}, \widehat{S}_{\beta}^{k}\right]_{+}
\end{aligned}
$$

при $k \neq j$,

$$
\begin{aligned}
& {\left[\widehat{S}_{\alpha}^{j}, \widehat{S}_{\beta}^{k}\right]_{-}=} \frac{\varphi_{\gamma}^{\hbar}\left(x_{j k}\right)}{\varphi_{0}^{\hbar}\left(x_{j k}\right)} \cdot \frac{\varphi_{\alpha}^{\hbar}\left(x_{k}\right) \varphi_{\beta}^{\hbar}\left(x_{j}\right)}{\varphi_{\alpha}^{\hbar}\left(x_{j}\right) \varphi_{\beta}^{\hbar}\left(x_{k}\right)}\left[\widehat{S}_{\alpha}^{k}, \widehat{S}_{\beta}^{j}\right]_{-}+ \\
&+i \varepsilon_{\alpha \beta \gamma} \frac{\varphi_{\alpha}^{\hbar}\left(x_{j k}\right)}{\varphi_{0}^{\hbar}\left(x_{j k}\right)} \cdot \frac{\varphi_{\gamma}^{\hbar}\left(x_{k}\right) \varphi_{0}^{\hbar}\left(x_{j}\right)}{\varphi_{\alpha}^{\hbar}\left(x_{j}\right) \varphi_{\beta}^{\hbar}\left(x_{k}\right)}\left[\widehat{S}_{\gamma}^{k}, \widehat{S}_{0}^{j}\right]_{+}- \\
&-i \varepsilon_{\alpha \beta \gamma} \frac{\varphi_{\beta}^{\hbar}\left(x_{j k}\right)}{\varphi_{0}^{\hbar}\left(x_{j k}\right)} \cdot \frac{\varphi_{\gamma}^{\hbar}\left(x_{j}\right) \varphi_{0}^{\hbar}\left(x_{k}\right)}{\varphi_{\alpha}^{\hbar}\left(x_{j}\right) \varphi_{\beta}^{\hbar}\left(x_{k}\right)}\left[\widehat{S}_{\gamma}^{j}, \widehat{S}_{0}^{k}\right]_{+}, \\
& {\left[\widehat{S}_{\alpha}^{j}, \widehat{S}_{0}^{k}\right]_{-}=\frac{\varphi_{\alpha}^{\hbar}\left(x_{j k}\right)}{\varphi_{0}^{\hbar}\left(x_{j k}\right)} \cdot \frac{\varphi_{\alpha}^{\hbar}\left(x_{k}\right) \varphi_{0}^{\hbar}\left(x_{j}\right)}{\varphi_{\alpha}^{\hbar}\left(x_{j}\right) \varphi_{0}^{\hbar}\left(x_{k}\right)}\left[\widehat{S}_{\alpha}^{k}, \widehat{S}_{0}^{j}\right]_{-}+} \\
& \quad+i \varepsilon_{\alpha \beta \gamma} \frac{\varphi_{\gamma}^{\hbar}\left(x_{j k}\right)}{\varphi_{0}^{\hbar}\left(x_{j k}\right)} \cdot \frac{\varphi_{\beta}^{\hbar}\left(x_{j}\right) \varphi_{\gamma}^{\hbar}\left(x_{k}\right)}{\varphi_{\alpha}^{\hbar}\left(x_{j}\right) \varphi_{0}^{\hbar}\left(x_{k}\right)}\left[\widehat{S}_{\beta}^{j}, \widehat{S}_{\gamma}^{k}\right]_{+}- \\
& \quad-i \varepsilon_{\alpha \beta \gamma} \frac{\varphi_{\beta}^{\hbar}\left(x_{j k}\right)}{\varphi_{0}^{\hbar}\left(x_{j k}\right)} \cdot \frac{\varphi_{\beta}^{\hbar}\left(x_{k}\right) \varphi_{\gamma}^{\hbar}\left(x_{j}\right)}{\varphi_{\alpha}^{\hbar}\left(x_{j}\right) \varphi_{0}^{\hbar}\left(x_{k}\right)}\left[\widehat{S}_{\beta}^{k}, \widehat{S}_{\gamma}^{j}\right]_{+}
\end{aligned}
$$

при $k=j$.

Выразим все коммутаторы через антикоммутаторы. Фактически мы имеем для скобок $\left[\widehat{S}_{\alpha}^{j}, \widehat{S}_{\beta}^{k}\right]_{-},\left[\widehat{S}_{\alpha}^{j}, \widehat{S}_{0}^{k}\right]_{-}$два добавочных уравнения $(j \leftrightarrow k)$. Решая систему из шести уравнений, получаем соотношения (4.13)-(4.16).

4.3. Квантовый детерминант. Докажем для группы $G L(2, \mathbb{C})$, что квантовый детерминант порождает центральные элементы перестановочной алгебры

$$
R_{12}\left(z_{1}, z_{2}\right) L_{1}\left(z_{1}\right) L_{2}\left(z_{2}\right)=L_{2}\left(z_{2}\right) L_{1}\left(z_{1}\right) R_{12}\left(z_{1}, z_{2}\right) .
$$

Рассмотрим случай классической алгебры (3.1)-(3.6). Чтобы доказать, что $\operatorname{det} L(z)$ в случае $G L(N, \mathbb{C})$ порождает функции Казимира пуассоновой структуры (3.1)-(3.6), рассмотрим каждую часть равенства

$$
\begin{aligned}
& \left\{L_{1}(z) \ldots L_{N}(z), L_{N+1}(w)\right\}= \\
& \quad=\left[L_{1}(z) \ldots L_{N}(z) L_{N+1}(w), r_{1, N+1}(z, w)+\cdots+r_{N, N+1}(z, w)\right],
\end{aligned}
$$

где $L_{i}$ и $r_{i k}, L_{i} \in \operatorname{End} V_{i}, r_{i k} \in \operatorname{End}\left(V_{i} \otimes V_{k}\right)$, как линейные операторы действуют на $\bigotimes_{i=1}^{N+1} V_{i}, V_{i} \cong \mathbb{C}^{N}$ - векторные пространства. Детерминант $\operatorname{det} L(z)$, очевидно, появляется на подпространстве $\left[\bigwedge_{i=1}^{N} V_{i}\right] \otimes V_{N+1}$. Правая часть равенства на этом подпространстве сводится к следующему выражению:

$$
\left[\operatorname{det} L(z) \cdot L_{N+1}(w), \operatorname{Tr}_{1} r_{1, N+1}(z, w)+\operatorname{Tr}_{N} r_{N, N+1}(z, w)\right] .
$$

Здесь следы $\operatorname{Tr}_{i}$ взяты над компонентами End $V_{i}$. Все они исчезают для $r$-матрицы (2.18). На этом заканчивается доказательство для случая классической алгебры. 
В квантовом случае детерминант заменяется квантовым детерминантом

$$
\operatorname{det}_{\hbar}=\operatorname{tr}\left(P^{-} \widehat{L}(z, \hbar) \otimes \widehat{L}(z+2 \hbar, \hbar)\right),
$$

где $P^{-}$- проекция на антисимметрическую часть тензорного произведения

$$
P^{-} a \otimes b=\frac{1}{2}(a \otimes b-b \otimes a) .
$$

Здесь мы рассмотрим только случай $(2 \times 2)$-матрицы. $R$-матрица

$$
R_{12}(z, w)=\sum_{a=0}^{3} \varphi_{a}^{\eta}(z-w) \sigma_{a} \otimes \sigma_{a}
$$

удовлетворяет следующим важным соотношениям:

$$
R_{12}(z, z+2 \hbar)=4 \frac{\vartheta^{\prime}(0)}{\vartheta(2 \hbar)} P^{-}, \quad P^{-}=\frac{1}{4}\left(1 \otimes 1-\sum_{\alpha=1}^{3} \sigma_{\alpha} \otimes \sigma_{\alpha}\right) .
$$

Рассмотрим произведение $L_{1}\left(z_{1}\right) L_{2}\left(z_{2}\right) L_{3}(w) \in V^{\otimes 3}$. Из уравнения Янга-Бакстера следует, что $R_{12} R_{13} R_{23} L_{1} L_{2} L_{3}=L_{3} L_{2} L_{1} R_{12} R_{13} R_{23}$. Положим $z_{2}=z_{1}+2 \hbar$, тогда $P_{12}^{-} R_{13} R_{23} L_{1} L_{2} L_{3}=L_{3} L_{2} L_{1} P_{12}^{-} R_{13} R_{23}$. Наиболее важно утверждение, что $P_{12}^{-} R_{13} R_{23} \sim P_{12}^{-} \otimes 1_{3}$, которое следует из прямых вычислений. Для упрощения можно использовать следующие тождества для $\alpha, \beta, \gamma \sim 1,2,3$ с точностью до циклических перестановок:

$$
-\varphi_{0}^{\hbar}(x) \varphi_{\gamma}(x-2 \hbar)+\varphi_{\gamma}^{\hbar}(x) \varphi_{0}(x-2 \hbar)+\varphi_{\beta}^{\hbar}(x) \varphi_{\alpha}(x-2 \hbar)+\varphi_{\alpha}^{\hbar}(x) \varphi_{\beta}(x-2 \hbar)=0 .
$$

Учитывая, что $\operatorname{Tr}_{12}\left(P_{12}^{-} L_{1} L_{2}\right)=\operatorname{Tr}_{12}\left(P_{12}^{-} L_{2} L_{1}\right)$, мы приходим к окончательному результату:

$$
\left[\operatorname{Tr}_{12}\left(P_{12}^{-} L_{1}(z-2 \hbar) L_{2}(z)\right), L_{3}(w)\right]=0
$$

4.4. Неоднородная алгебра и уравнение отражения. Рассмотрим случай ранга $N=2$ с четырьмя отмеченными точками $n=4$. Пусть сначала отмеченные точки находятся в точке $z=0$ и в полупериодах $\Sigma_{\tau}$

$$
x_{0}=0, \quad x_{1}=\frac{\tau}{2}=\omega_{2}, \quad x_{2}=\frac{1+\tau}{2}=\omega_{1}+\omega_{2}, \quad x_{3}=\frac{1}{2}=\omega_{1} .
$$

Предположим, что

$$
S_{\alpha}^{j}=\delta_{\alpha}^{j} \tilde{\nu}_{\alpha}, \quad j=1,2,3,
$$

где $S_{\alpha}^{0}=S_{\alpha}$ произвольны. Такой выбор основан на редукции $L(z) L(-z)=\operatorname{det} L(z) \cdot I$.

Пусть $R^{-}$- квантовая вертексная $R$-матрица, которая возникает в XYZ-модели. Введем также $R^{+}$-матрицу

$$
R^{ \pm}(z, w)=\sum_{a=0}^{3} \varphi_{a}^{\hbar / 2}(z \pm w) \sigma_{a} \otimes \sigma_{a} .
$$

Определим квантовый оператор Лакса

$$
\widehat{L}(z)=\widehat{S}_{0} \phi^{\hbar}(z) \sigma_{0}+\sum_{\alpha}\left(\widehat{S}_{\alpha} \varphi_{\alpha}^{\hbar}(z)+\tilde{\nu}_{\alpha} \varphi_{\alpha}^{\hbar}\left(z-\omega_{\alpha}\right)\right) \sigma_{\alpha} .
$$


ПреДЛОжЕНИЕ 8. Оператор Лакса (4.25) удовлетворяет квантовому уравнению отражения

$$
R^{-}(z, w) \widehat{L}_{1}(z) R^{+}(z, w) \widehat{L}_{2}(w)=\widehat{L}_{2}(w) R^{+}(z, w) \widehat{L}_{1}(z) R^{-}(z, w)
$$

если его компоненты $S_{a}$ порождают ассочиативную алгебру с соотношениями

$$
\begin{gathered}
{\left[\tilde{\nu}_{\alpha}, \tilde{\nu}_{\beta}\right]=0, \quad\left[\tilde{\nu}_{\alpha}, \widehat{S}_{a}\right]=0,} \\
i\left[\widehat{S}_{0}, \widehat{S}_{\alpha}\right]_{+}=\left[\widehat{S}_{\beta}, \widehat{S}_{\gamma}\right], \\
{\left[\widehat{S}_{\gamma}, \widehat{S}_{0}\right]=i \frac{K_{\beta}-K_{\alpha}}{K_{\gamma}}\left[\widehat{S}_{\alpha}, \widehat{S}_{\beta}\right]_{+}-2 i \frac{1}{K_{\gamma}}\left(\tilde{\nu}_{\alpha} \rho_{\alpha} \widehat{S}_{\beta}-\tilde{\nu}_{\beta} \rho_{\beta} \widehat{S}_{\alpha}\right),}
\end{gathered}
$$

где

$$
K_{\alpha}=E_{1}(\hbar+\check{\alpha})-E_{1}(\hbar)-E_{1}(\check{\alpha}), \quad \rho_{\alpha}=-\exp \left(-2 \pi i \check{\alpha} \partial_{\tau} \check{\alpha}\right) \phi(\check{\alpha}+\hbar,-\check{\alpha}) .
$$

Доказательство основано на прямом вычислении левой и правой частей (4.26) (см. подробнее в [9]). Если в соотношениях (4.27)-(4.29) все $\nu_{\alpha}=0$, то ассоциативная алгебра совпадает с алгеброй Склянина. Поэтому алгебра, определяемая соотношениями (4.27)-(4.29), является трехпараметрической деформацией алгебры Склянина.

Элементы

$$
C_{1}=\widehat{S}_{0}^{2}+\sum_{\alpha} \widehat{S}_{\alpha}^{2}, \quad C_{2}=\sum_{\alpha} \widehat{S}_{\alpha}^{2} K_{\alpha}\left(K_{\alpha}-K_{\beta}-K_{\gamma}\right)+2 \tilde{\nu}_{\alpha} \rho_{\alpha} K_{\alpha} \widehat{S}_{\alpha}
$$

принадлежат центру обобщенной алгебры Склянина (4.27), (4.28). Они являются коэффициентами в разложении квантового детерминанта

$$
\operatorname{det}_{\hbar}=\operatorname{tr}\left(P^{-\widehat{L}}(z, \hbar) \otimes \widehat{L}(z+2 \hbar, \hbar)\right) .
$$

ПРИЛОЖЕНИЕ А

\section{Эллиптические функции}

Положим $q=e^{2 \pi i \tau}$, где $\tau$ - модулярный параметр эллиптической кривой $E_{\tau}$. Базисным элементом является тета-функция:

$$
\vartheta(z \mid \tau)=q^{1 / 8} \sum_{n \in \mathbb{Z}}(-1)^{n} \mathbf{e}\left(\frac{1}{2} n(n+1) \tau+n z\right), \quad \mathbf{e}(x)=e^{2 \pi i x} .
$$

Введем функции Эйзенштейна

$$
E_{1}(z \mid \tau)=\partial_{z} \ln \vartheta(z \mid \tau), \quad E_{1}(z \mid \tau) \sim \frac{1}{z}-2 \eta_{1} z
$$

где

$$
\eta_{1}(\tau)=\frac{24}{2 \pi i} \frac{\eta^{\prime}(\tau)}{\eta(\tau)}, \quad \eta(\tau)=q^{1 / 24} \prod_{n>0}\left(1-q^{n}\right),
$$

- функция Дедекинда и

$$
E_{2}(z \mid \tau)=-\partial_{z} E_{1}(z \mid \tau)=\partial_{z}^{2} \ln \vartheta(z \mid \tau), \quad E_{2}(z \mid \tau) \sim \frac{1}{z^{2}}+2 \eta_{1} .
$$


Справедливы следующие соотношения на функции Вейерштрасса:

$$
\zeta(z, \tau)=E_{1}(z, \tau)+2 \eta_{1}(\tau) z, \quad \wp(z, \tau)=E_{2}(z, \tau)-2 \eta_{1}(\tau) .
$$

Введем высшие функции Эйзенштейна

$$
E_{j}(z)=\frac{(-1)^{j}}{(j-1) !} \partial^{(j-2)} E_{2}(z), \quad j>2 .
$$

Пусть

$$
\phi(u, z)=\frac{\vartheta(u+z) \vartheta^{\prime}(0)}{\vartheta(u) \vartheta(z)} .
$$

Тогда $\phi(u, z)=\phi(z, u), \phi(-u,-z)=-\phi(u, z)$. Функция $\phi$ имеет полюс в точке $z=0$ и

$$
\begin{aligned}
\phi(u, z)= & \frac{1}{z}+E_{1}(u)+\frac{z}{2}\left(E_{1}^{2}(u)-\wp(u)\right)+\cdots, \\
\partial_{u} \phi(u, z)= & \phi(u, z)\left(E_{1}(u+z)-E_{1}(u)\right), \\
\partial_{z} \phi(u, z)= & \phi(u, z)\left(E_{1}(u+z)-E_{1}(z)\right), \\
& \lim _{z \rightarrow 0} \ln \partial_{u} \phi(u, z)=-E_{2}(u) .
\end{aligned}
$$

Функция $\phi$ удовлетворяет уравнению теплопроводности

$$
\partial_{\tau} \phi(u, w)-\frac{1}{2 \pi i} \partial_{u} \partial_{w} \phi(u, w)=0 .
$$

Квазипериоды для введенных функций определяются следующим образом:

$$
\begin{array}{rlrl}
\vartheta(z+1) & =-\vartheta(z), & \vartheta(z+\tau) & =-q^{-1 / 2} e^{-2 \pi i z} \vartheta(z), \\
E_{1}(z+1) & =E_{1}(z), & E_{1}(z+\tau) & =E_{1}(z)-2 \pi i, \\
E_{2}(z+1) & =E_{2}(z), & E_{2}(z+\tau) & =E_{2}(z), \\
\phi(u, z+1) & =\phi(u, z), & \phi(u, z+\tau) & =e^{-2 \pi i u} \phi(u, z), \\
\partial_{u} \phi(u, z+1)=\partial_{u} \phi(u, z), & \partial_{u} \phi(u, z+\tau) & =e^{-2 \pi i u} \partial_{u} \phi(u, z)-2 \pi i \phi(u, z) .
\end{array}
$$

Справедливо тождество Фэя:

$$
\phi\left(u_{1}, z_{1}\right) \phi\left(u_{2}, z_{2}\right)-\phi\left(u_{1}+u_{2}, z_{1}\right) \phi\left(u_{2}, z_{2}-z_{1}\right)-\phi\left(u_{1}+u_{2}, z_{2}\right) \phi\left(u_{1}, z_{1}-z_{2}\right)=0 \text {. }
$$

Из (А.7) и (А.8) получаем:

$$
\phi\left(u_{1}, z\right) \phi\left(u_{2}, z\right)=\phi\left(u_{1}+u_{2}, z\right)\left(E_{1}\left(u_{1}\right)+E_{1}\left(u_{2}\right)-E_{1}\left(u_{1}+u_{2}+z\right)+E_{1}(z)\right) .
$$

Частными случаями тождества (А.8) являются следующие функциональные уравнения:

$$
\begin{aligned}
\phi(u, z) \partial_{v} \phi(v, z)-\phi(v, z) \partial_{u} \phi(u, z)= & \left(E_{2}(v)-E_{2}(u)\right) \phi(u+v, z), \\
\phi\left(u, z_{1}\right) \phi\left(-u, z_{2}\right)= & \phi\left(u, z_{1}-z_{2}\right)\left(-E_{1}\left(z_{1}\right)+E_{1}\left(z_{2}\right)-\right. \\
& \left.-E_{1}(u)+E_{1}\left(u+z_{1}-z_{2}\right)\right)=\quad(\mathrm{A} .10) \\
= & \phi\left(u, z_{1}-z_{2}\right)\left(-E_{1}\left(z_{1}\right)+E_{1}\left(z_{2}\right)+\partial_{u} \phi\left(u, z_{2}-z_{1}\right)\right),
\end{aligned}
$$




$$
\begin{gathered}
\phi(u, z) \phi(-u, z)=E_{2}(z)-E_{2}(u), \\
\phi(v, z-w) \phi\left(u_{1}-v, z\right) \phi\left(u_{2}+v, w\right)-\phi\left(u_{1}-u_{2}-v, z-w\right) \phi\left(u_{2}+v, z\right) \phi\left(u_{1}-v, w\right)= \\
=\phi\left(u_{1}, z\right) \phi\left(u_{2}, w\right) f\left(u_{1}, u_{2}, v\right),
\end{gathered}
$$

где

$$
f\left(u_{1}, u_{2}, v\right)=E_{1}(v)-E_{1}\left(u_{1}-u_{2}-v\right)+E_{1}\left(u_{1}-v\right)-E_{1}\left(u_{2}+v\right) .
$$

Функцию (А.12) можно переписать в виде

$$
f\left(u_{1}, u_{2}, v\right)=-\frac{\vartheta^{\prime}(0) \vartheta\left(u_{1}\right) \vartheta\left(u_{2}\right) \vartheta\left(u_{2}-u_{1}+2 v\right)}{\vartheta\left(u_{1}-v\right) \vartheta\left(u_{2}+v\right) \vartheta\left(u_{2}-u_{1}+v\right) \vartheta(v)} .
$$

Используя (А.1), (А.2), (А.5) можно получить из (А.11) некоторые важные специальные случаи. В первом случае при $v=u_{1}$ (или $v=-u_{2}$ ) получим тождество Фэя (А.8). Во втором случае при $u_{1}=0$ (или $u_{2}=u$ ) имеем

$$
\begin{gathered}
\phi(v, z-w) \phi(-v, z) \phi(u+v, w)-\phi(-u-v, z-w) \phi(u+v, z) \phi(-v, w)= \\
=\phi\left(u_{1}, z\right)\left(E_{2}(u+v)-E_{2}(v)\right) .
\end{gathered}
$$

Если $u_{2} \rightarrow-v$, то соотношение (А.11) при $u_{1}=\alpha, u_{2}=\beta$ в первом нетривиальном порядке принимает вид

$$
\begin{gathered}
\phi(-\beta, z-w) E_{1}(w) \phi(\alpha+\beta, z)-\phi(\alpha, z-w) E_{1}(z) \phi(\alpha+\beta, w)= \\
=\phi(\alpha, z) \phi(\beta, w)\left(E_{1}(\alpha)+E_{1}(\beta)-E_{1}(\alpha+\beta)\right) .
\end{gathered}
$$

ПРИЛОЖЕНИЕ Б

\section{Алгебра Ли $\operatorname{sl}(N, \mathbb{C})$ и эллиптические функции}

Введем следующие обозначения:

$$
\begin{gathered}
\mathbf{e}_{N}(z)=e^{2 \pi i z / N}, \\
Q=\operatorname{diag}\left(\mathbf{e}_{N}(1), \ldots, \mathbf{e}_{N}(m), \ldots, 1\right), \\
\Lambda=\delta_{j, j+1}, \quad j=1, \ldots, N \bmod N .
\end{gathered}
$$

Пусть

$$
\mathbb{Z}_{N}^{(2)}=(\mathbb{Z} / N \mathbb{Z} \oplus \mathbb{Z} / N \mathbb{Z}), \quad \widetilde{\mathbb{Z}}_{N}^{(2)}=\mathbb{Z}_{N}^{(2)} \backslash(0,0),
$$

- двумерные решетки порядка $N^{2}$ и $N^{2}-1$ соответственно. Матрицы $Q^{a_{1}} \Lambda^{a_{2}}, a=$ $\left(a_{1}, a_{2}\right) \in \mathbb{Z}_{N}^{(2)}$, индуцируют базис в группе $G L(N, \mathbb{C})$, а $Q^{\alpha_{1}} \Lambda^{\alpha_{2}}, \alpha=\left(\alpha_{1}, \alpha_{2}\right) \in \widetilde{\mathbb{Z}}_{N}^{(2)}$, индуцируют базис в алгебре Ли $\operatorname{sl}(N, \mathbb{C})$. Подробнее рассмотрим представление $\mathbb{Z}_{N}^{(2)}$ в $G L(N, \mathbb{C})$ :

$$
\begin{aligned}
a \rightarrow T_{a} & =\frac{N}{2 \pi i} \mathbf{e}_{N}\left(\frac{a_{1} a_{2}}{2}\right) Q^{a_{1}} \Lambda^{a_{2}}, \\
T_{a} T_{b} & =\frac{N}{2 \pi i} \mathbf{e}_{N}\left(-\frac{a \times b}{2}\right) T_{a+b}, \quad a \times b=a_{1} b_{2}-a_{2} b_{1},
\end{aligned}
$$


где $N \mathbf{e}_{N}(-a \times b / 2) /(2 \pi i)$ - нетривиальный 2 -коцикл в $H^{2}\left(\mathbb{Z}_{N}^{(2)}, \mathbb{Z}_{2 N}\right)$. Матрицы $T_{\alpha}$, $\alpha \in \widetilde{\mathbb{Z}}_{N}^{(2)}$, индуцируют базис в $\operatorname{sl}(N, \mathbb{C})$. Из (Б.2) следует, что

$$
\left[T_{\alpha}, T_{\beta}\right]=\mathbf{C}(\alpha, \beta) T_{\alpha+\beta}
$$

где $\mathbf{C}(\alpha, \beta)=(N / \pi) \sin (\pi(\alpha \times \beta) / N)$ - структурные константы в $\operatorname{sl}(N, \mathbb{C})$.

При $N=2$ базис $T_{\alpha}$ пропорционален базису матриц Паули:

$$
T_{(1,0)}=\frac{1}{\pi i} \sigma_{3}, \quad T_{(0,1)}=\frac{1}{\pi i} \sigma_{1}, \quad T_{(1,1)}=\frac{1}{\pi i} \sigma_{2} .
$$

Коалгебра Ли $\mathfrak{g}^{*}=\operatorname{sl}(N, \mathbb{C})$ имеет дуальный базис

$$
\mathfrak{g}^{*}=\left\{\mathbf{S}=\sum_{\widetilde{\mathbb{Z}}_{N}^{(2)}} S_{\gamma} t^{\gamma}\right\}, \quad t^{\gamma}=\frac{2 \pi i}{N^{2}} T_{-\gamma}, \quad\left\langle T_{\alpha} t^{\beta}\right\rangle=\delta_{\alpha}^{-\beta}
$$

Из (Б.3) следует, что $\mathfrak{g}^{*}$ является многообразием Пуассона, оснащенным скобками

$$
\left\{S_{\alpha}, S_{\beta}\right\}=\mathbf{C}(\alpha, \beta) S_{\alpha+\beta} .
$$

Коприсоединенное действие в этих базисах имеет вид

$$
\operatorname{ad}_{T_{\alpha}}^{*} t^{\beta}=\mathbf{C}(\alpha, \beta) t^{\alpha+\beta}
$$

Пусть $\breve{\gamma}=\left(\gamma_{1}+\gamma_{2} \tau\right) / N$. Введем константы на $\widetilde{\mathbb{Z}}^{(2)}$ :

$$
\vartheta(\breve{\gamma})=\vartheta\left(\frac{\gamma_{1}+\gamma_{2} \tau}{N}\right), \quad E_{1}(\breve{\gamma})=E_{1}\left(\frac{\gamma_{1}+\gamma_{2} \tau}{N}\right), \quad E_{2}(\breve{\gamma})=E_{2}\left(\frac{\gamma_{1}+\gamma_{2} \tau}{N}\right)
$$

и функции

$$
\begin{aligned}
\phi_{\gamma}(z) & =\phi(\breve{\gamma}, z) \\
\varphi_{\gamma}(z) & =\mathbf{e}_{N}\left(\gamma_{2} z\right) \phi_{\gamma}(z) \\
\varphi_{\gamma, \eta}(z) & =\mathbf{e}_{N}\left(\gamma_{2} z\right) \phi\left(\eta+\frac{\gamma_{1}+\gamma_{2} \tau}{N}, z\right) .
\end{aligned}
$$

Эти функции имеют следующие квазипериоды:

$$
\begin{aligned}
\varphi_{\gamma}(z+1) & =\mathbf{e}_{N}\left(\gamma_{2}\right) \varphi_{\gamma}(z), & \varphi_{\gamma}(z+\tau) & =\mathbf{e}_{N}\left(-\gamma_{1}\right) \varphi_{\gamma}(z), \\
\varphi_{\gamma, \eta}(z+1) & =\mathbf{e}_{N}\left(\gamma_{2}\right) \varphi_{\gamma, \eta}(z), & \varphi_{\gamma, \eta}(z+\tau) & =\mathbf{e}_{N}\left(-\gamma_{1}-\eta\right) \varphi_{\gamma, \eta}(z) .
\end{aligned}
$$

Важные соотношения для этих функций имеют вид

$$
\begin{aligned}
\frac{\varphi_{\eta}\left(z_{1}-z_{2}\right)}{\varphi_{\eta}\left(z_{1}\right)} & =\frac{1}{\varphi_{\eta}\left(z_{2}\right)}\left(E_{1}\left(z_{2}\right)+E_{1}(\eta)+E_{1}\left(z_{1}-z_{2}\right)-E_{1}\left(z_{1}+\eta\right)\right), \\
\frac{\varphi_{\alpha, \eta}\left(z_{1}-z_{2}\right)}{\varphi_{\eta}\left(z_{1}\right)} & =\frac{1}{\varphi_{\eta}\left(z_{2}\right)} \varphi_{\alpha}\left(z_{1}-z_{2}\right)+\frac{\varphi_{-\alpha}\left(z_{2}\right)}{\varphi_{\eta}\left(z_{2}\right) \varphi_{\alpha}(\eta)} \varphi_{\alpha}\left(z_{1}+\eta\right), \\
\frac{\varphi_{\alpha, \eta}(z)}{\varphi_{\eta}(z)} & =\frac{\varphi_{\alpha}(z+\eta)}{\varphi_{\alpha}(\eta)} .
\end{aligned}
$$


Другое важное соотношение в случае $N=2$ :

$$
k_{\gamma} f^{\hbar}(\gamma, \alpha, 0)=J_{\gamma}=E_{2}(\gamma+\hbar)-E_{2}(\hbar),
$$

где

$$
k_{\gamma}=E_{1}(\gamma+\hbar)-E_{1}(\gamma)-E_{1}(\hbar)
$$

Дадим короткие пояснения к этой формуле. Из (А.11), (А.12) имеем:

$\left(E_{1}(\gamma+\hbar)-E_{1}(\gamma)-E_{1}(\hbar)\right)\left(E_{1}(\alpha+\hbar)+E_{1}(-\beta+\hbar)-E_{1}(\gamma+\hbar)-E_{1}(\hbar)\right)=E_{2}(\gamma+\hbar)-E_{2}(\hbar)$,

где $\alpha-\beta=\gamma$. Функции в правой и в левой частях имеют совпадающие полюсы $(\hbar=0, \hbar=-\gamma)$ и нули $(\hbar=-\gamma / 2)$, поэтому эти функции эквивалентны.

Определим функцию

$$
f_{\gamma}(z)=\left.\mathbf{e}_{N}\left(\gamma_{2} z\right) \partial_{u} \phi(u, z)\right|_{u=\breve{\gamma}}=\varphi_{\gamma}(z)\left(E_{1}(\breve{\gamma}+z)-E_{1}(\breve{\gamma})\right)
$$

Из (А.6) следует, что

$$
\begin{aligned}
f_{\gamma}(z) & =\varphi_{\gamma}(z)\left(E_{1}(\breve{\gamma}+z)-E_{1}(\breve{\gamma})\right) \\
f(\alpha, \beta, \gamma) & =E_{1}(\breve{\gamma})-E_{1}(\breve{\alpha}-\breve{\beta}-\breve{\gamma})+E_{1}(\breve{\alpha}-\breve{\gamma})-E_{1}(\breve{\beta}-\breve{\gamma})
\end{aligned}
$$

(см. (A.12)).

Из (А.4) вытекает, что

$$
\begin{aligned}
\varphi_{\gamma}(z+1) & =\mathbf{e}_{N}\left(\gamma_{2}\right) \varphi_{\gamma}(z), & \varphi_{\gamma}(z+\tau) & =\mathbf{e}_{N}\left(-\gamma_{1}\right) \varphi_{\gamma}(z), \\
f_{\gamma}(z+1) & =\mathbf{e}_{N}\left(\gamma_{2}\right) f_{\gamma}(z), & f_{\gamma}(z+\tau) & =\mathbf{e}_{N}\left(-\gamma_{1}\right) f_{\gamma}(z)-2 \pi i \varphi_{\gamma}(z) .
\end{aligned}
$$

Модификацией (А.11) является соотношение

$$
\varphi_{\gamma}\left(z-x_{j}\right) \varphi_{-\gamma}\left(z-x_{k}\right)=\varphi_{\gamma}\left(x_{k}-x_{j}\right)\left(E_{1}\left(z-x_{k}\right)-E_{1}\left(z-x_{j}\right)\right)-f_{\gamma}\left(x_{k}-x_{j}\right) .
$$

Благодарности. А. Левин и А. Зотов благодарны за гостеприимство Институту Макса Планка в Бонне, где эта работа была подготовлена. Работа выполнена при частичной поддержке РФФИ (грант № 06-02-17381, № 06-01-92054-KЕ) и Программы поддержки ведущих научных школ (грант № НШ-8065.2006.2). Работа А. Зотова была также поддержана фондом "Династия".

\section{Список литературы}

[1] А. А. Белавин, В. Г. Дринфельд, Функи. анализ и его прил., 16:3 (1982), 1-29.

[2] А. Белавин, Функи. анализ и его прил., 14:4 (1980), 18-26.

[3] Е. Склянин, Функи. анализ и его прил., 16:4 (1982), 27-34.

[4] А. В. Одесский, Б.Л. Фейгин, Функи. анализ и его прил., 23:3 (1989), 45-54.

[5] K. Takasaki, Lett. Math. Phys., 44:2 (1998), 143-156; arXiv: hep-th/9711058.

[6] Yu. Chernyakov, A. Levin, M. Olshanetsky, A. Zotov, J. Phys. A, 39:39 (2006), 12083-12101; arXiv: nlin.SI/0602043.

[7] H. Braden, V. Dolgushev, M. Olshanetsky, A. Zotov, J. Phys. A, 36:25 (2003), 6979-7000.

[8] B. Khesin, A. Levin, M. Olshanetsky, Comm. Math. Phys., 250:3 (2004), 581-612.

[9] A. Levin, M. Olshanetsky, A. Zotov, Comm. Math. Phys., 268:1 (2006), 67-103; arXiv: math.QA/0508058. 